\title{
Dendritic Compartment and Neuronal Output Mode Determine Pathway-Specific Long-Term Potentiation in the Piriform Cortex
}

\author{
Friedrich W. Johenning, ${ }^{1}$ Prateep S. Beed, ${ }^{1}$ Thorsten Trimbuch, ${ }^{2}$ Michael H. K. Bendels,${ }^{1,3}$ Jochen Winterer, ${ }^{1}$ \\ and Dietmar Schmitz ${ }^{1,4}$ \\ ${ }^{1}$ Neuroscience Research Center, ${ }^{2}$ Institute for Cell Biology and Neurobiology, Center for Anatomy, and ${ }^{3}$ Neurophysics Group, Department of Neurology, \\ Charité-Universitätsmedizin Berlin, 10117 Berlin, Germany, and ${ }^{4}$ Bernstein Center for Computational Neuroscience Berlin, 10115 Berlin, Germany
}

The apical dendrite of layer 2/3 pyramidal cells in the piriform cortex receives two spatially distinct inputs: one projecting onto the distal apical dendrite in sensory layer 1a, the other targeting the proximal apical dendrite in layer 1b. We observe an expression gradient of A-type $\mathrm{K}^{+}$channels that weakens the backpropagating action potential-mediated depolarization in layer la compared with layer $1 \mathrm{~b}$. We find that the pairing of presynaptic and postsynaptic firing leads to significantly smaller $\mathrm{Ca}^{2+}$ signals in the distal dendritic spines in layer 1a compared with the proximal spines in layer $1 \mathrm{~b}$. The consequence is a selective failure to induce long-term potentiation (LTP) in layer 1a, which can be rescued by pharmacological enhancement of action potential backpropagation. In contrast, LTP induction by pairing presynaptic and postsynaptic firing is possible in layer $1 \mathrm{~b}$ but requires bursting of the postsynaptic cell. This output mode strongly depends on the balance of excitation and inhibition in the piriform cortex. We show, on the single-spine level, how the plasticity of functionally distinct synapses is gated by the intrinsic electrical properties of piriform cortex layer 2 pyramidal cell dendrites and the cellular output mode.

\section{Introduction}

Two spatially distinct pathways project onto the apical dendrites of layer $2 / 3$ pyramidal cells in layer 1 of the piriform cortex. Direct synaptic inputs from mitral cells in the olfactory bulb project onto the distal portion of the dendrite, constituting the sensory layer 1a. Associational synapses from within the piriform cortex and other brain areas cluster in the proximal layer $1 \mathrm{~b}$ (Neville and Haberly, 2004). This laminar structure allows a clear spatial distinction between sensory and associative inputs. A key functional difference between the two synaptic inputs is their plasticity. NMDA receptor (NMDAR)-dependent long-term potentiation (LTP) of synaptic contacts is much more pronounced in the proximal associative layer $1 \mathrm{~b}$ than in the distal sensory layer $1 \mathrm{a}$ (Jung et al., 1990a,b; Kanter and Haberly, 1990; Franks and Isaacson, 2005; Poo and Isaacson, 2007). So far, mechanisms determining the weakness of layer 1a LTP have only been found on the level of LTP expression (Franks and Isaacson, 2005). The induction of LTP by pairing EPSPs with subsequent action potentials

Received June 8, 2009; revised July 24, 2009; accepted Aug. 5, 2009.

This work was supported by Deutsche Forschungsgemeinschaft Grants EXC 257, GRK 1123, SFB 618, and SFB 665 and the Bundesministerium für Bildung und Forschung (Bernstein Center for Computational Neuroscience Berlin). P.S.B. is a member of GRK 1123 (Berlin, Germany). We thank Genela Morris, Jörg Breustedt, Knut Holthoff, and Sarah Shoichet for critically reading this manuscript and Susanne Walden, Anke Schönherr, and Denis Lajkó for excellent technical assistance.

Correspondence should be addressed to either Dietmar Schmitz or Friedrich W. Johenning, Neurowissenschaftliches Forschungszentrum, Charité-Universitätsmedizin Berlin, Charitéplatz 1, 10117 Berlin, Germany. E-mail: dietmar.schmitz@charite.de or friedrich.johenning@charite.de.

DOI:10.1523/JNEUROSCI.2672-09.2009

Copyright $\odot 2009$ Society for Neuroscience $\quad$ 0270-6474/09/2913649-13\$15.00/0
(APs) is reduced in the distal dendrites in neocortical pyramidal cells and in the CA1 pyramidal cells in the hippocampus. In these neurons, the attenuated distal propagation of backpropagating APs (bAPs), mediated by the intrinsic electrical properties of the dendritic tree, weakens distal LTP induction (Golding et al., 2002; Letzkus et al., 2006; Sjöström and Häusser, 2006).

In area CA1 within the hippocampal formation and also the neocortex, LTP can be induced by pairing EPSPs with bursts of APs (Pike et al., 1999; Kampa et al., 2006; Letzkus et al., 2006; Nevian and Sakmann, 2006). It has been demonstrated by Suzuki and Bekkers that intrinsic AP bursting during suprathreshold depolarization is a key feature of piriform cortex pyramidal cells. In the same study, short bursts with high instantaneous firing frequencies of up to $150 \mathrm{~Hz}$ were evoked by strong stimulation of sensory or associative pathways with typical sensory input patterns (Suzuki and Bekkers, 2006). During sniffing, short AP bursts with instantaneous firing rates above $100 \mathrm{~Hz}$ have also been observed in intracellular recordings in vivo (Wilson, 1998). Recently, it has been shown in vitro that selective stimulation of the sensory lateral olfactory tract (LOT) input to layer 1a results in prominent feedforward inhibition, limiting the output mode to single action potentials. A reduction in inhibitory drive turned these single APs into short bursts (Luna and Schoppa, 2008). Bursting and single-spike firing modes of pyramidal cells in the piriform cortex appear tightly controlled by the ratio of excitation (synaptic and intrinsic) to inhibition.

During LTP induction, $\mathrm{Ca}^{2+}$ signals in postsynaptic spines are a key determinant of plasticity outcome (Nevian and Sakmann, 2006) (for review, see Sjöström et al., 2008). Here, we analyze $\mathrm{Ca}^{2+}$ sig- 
nals in proximal and distal dendritic segments within pyramidal neurons of the piriform cortex. We demonstrate that the laminar distribution of different synaptic inputs is a prerequisite for differences in synaptic plasticity induction based on the electrical compartmentalization of the dendritic tree. We further propose that LTP induction in the plasticity-competent layer $1 \mathrm{~b}$ synapses is gated by the neuronal output mode (single APs vs bursting).

\section{Materials and Methods}

\section{Electrophysiology}

Preparation. Coronal slices of the anterior piriform cortex were prepared from Wistar rats [aged postnatal day 19 (P19) to P25]. All procedures were performed in accordance with national and international guidelines. Animals were anesthetized and decapitated. The brains were quickly removed into ice-cold artificial CSF (ACSF) containing the following (in mM): $87 \mathrm{NaCl}, 26 \mathrm{NaHCO}_{3}, 25$ glucose, $2.5 \mathrm{KCl}, 7 \mathrm{MgCl}_{2}, 1.25$ $\mathrm{NaH} 2 \mathrm{PO}_{4}, 0.5 \mathrm{CaCl}_{2}$, and 75 sucrose, $\mathrm{pH}$ 7.4. Tissue blocks were mounted on a vibratome (VT 1200; Leica Microsystems), cut at $300 \mu \mathrm{m}$ thickness, and incubated at $35^{\circ} \mathrm{C}$ for $30 \mathrm{~min}$. The slices were then transferred to ACSF containing the following (in $\mathrm{mm}$ ): $119 \mathrm{NaCl}, 26 \mathrm{NaHCO}_{3}$, 10 glucose, $2.5 \mathrm{KCl}, 2.5 \mathrm{CaCl}_{2}, 1 \mathrm{MgSO}_{4}$, and $1 \mathrm{NaH}_{2} \mathrm{PO}_{4}$. For LTP experiments, $2 \mu \mathrm{M}$ gabazine was added. For the 4-AP experiments looking at bAP-mediated $\mathrm{Ca}^{2+}, 25 \mu \mathrm{M}$ NBQX, $50 \mu \mathrm{M} \mathrm{D}-\mathrm{APV}$, and $2 \mu \mathrm{M}$ gabazine were added to the ACSF. LTP experiments in the presence of 4 -AP were performed in $2 \mu \mathrm{M}$ gabazine and $100 \mathrm{~nm}$ NBQX. The slices were stored at room temperature in a submerged chamber for $1-5 \mathrm{~h}$ before being transferred to the recording chamber.

Whole-cell recordings. Whole-cell voltage-clamp and current-clamp recordings were performed with an Axopatch 700B Amplifier (Molecular Devices) at $28-30^{\circ} \mathrm{C}$. Data were digitized at $5 \mathrm{kHz}$ and low-pass filtered at $2 \mathrm{kHz}$. For $\mathrm{Ca}^{2+}$ imaging experiments, patch electrodes [electrode resistances ranging from 3 to $6 \mathrm{M} \Omega$ were filled with the following (in $\mathrm{mM}$ ): 115 K-gluconate, $20 \mathrm{KCl}, 10 \mathrm{HEPES}, 4 \mathrm{MgATP}, 0.3 \mathrm{Na}-\mathrm{GTP}$, and 10 phosphocreatine, pH 7.3 with $\mathrm{KOH}$ ]. Oregon-Green BAPTA-1 (OGB1) was added at $100 \mu \mathrm{m}$ and Oregon-Green BAPTA-6F (OGB6F) at $200 \mu \mathrm{M}$. For LTP experiments, the intracellular solution consisted of the following (in mM): $130 \mathrm{~K}$-gluconate, $20 \mathrm{KCl}, 10 \mathrm{HEPES}, 4 \mathrm{MgATP}, 0.3 \mathrm{Na}-\mathrm{GTP}$, and 10 phosphocreatine, $\mathrm{pH} 7.4$ with $\mathrm{KOH}$. Initial access resistances were in the range of 10 and $20 \mathrm{M} \Omega$ after breakthrough and not allowed to vary $>20 \%$ during the course of the experiment in the voltage-clamp mode. No access resistance compensation was used.

APs were induced with $2 \mathrm{~ms}$ pulses at $1-3 \mathrm{nA}$. Synaptic stimulation was performed with patch pipettes filled with ACSF. For LTP measurements, the pipette was placed at the layer 2-layer $1 \mathrm{~b}$ border for layer $1 \mathrm{~b}$ stimulation and at the LOT-layer la border for layer la stimulation (see Fig. $6 \mathrm{~A}$ ). The test stimulus was applied in the voltage-clamp mode, the amplitude was between 50 and $200 \mathrm{pA}$. For LTP induction, the amplifier was switched to current clamp. Stimulus artifacts are clipped in all figures.

\section{Morphological reconstruction}

Slices with biocytin-filled cells were fixed in $0.1 \mathrm{~mm}$ phosphate buffer, $\mathrm{pH}$ 7.4, containing $4 \%$ paraformaldehyde (PFA), for $24-48 \mathrm{~h}$. The filled neurons were visualized by incubating sections in avidin-biotinconjugated horseradish peroxidase (ABC; Vector Laboratories) and reacting them with diaminobenzidine and hydrogen peroxide. Sections were then dehydrated and embedded on glass slides. Subsequently, the cells were reconstructed with the aid of a Neurolucida $3 \mathrm{D}$ reconstruction system (MicroBrightField).

\section{Immunohistochemistry}

For immunohistochemistry, P21 rats were anesthetized ( $0.5 \%$ ketamine, i.p.) and transcardially perfused with $4 \%$ PFA. Brains were postfixed overnight in $4 \%$ PFA and cut on a vibratome. Sections at $50 \mu \mathrm{m}$ were blocked for $1 \mathrm{~h}$ (5\% normal goat serum and $0.1 \%$ Triton X-100) before overnight incubation with antibodies against rabbit Kv4.2/4.3 (H-225) (1:200; Santa Cruz Biotechnology) and mouse MAP2 (HM-2) (1:1000; Sigma). Sections were immunostained with Alexa-labeled secondary antibodies (Invitrogen). Images were obtained with a Leica Microsystems confocal laser-scanning microscope (TCS SL). For quantification of Kv4.2/4.3 expression in the piriform cortex layers $1 \mathrm{a}$ and $1 \mathrm{~b}$, randomly chosen $50 \times 50 \mu \mathrm{m}$ subregions aligned to follow the main vertical dendritic axis were scanned with a $63 \times$ oil objective (zoom 5 , same configurations in both areas) and quantified using MetaMorph (version 6.2r6). The number of Kv4.2/4.3 puncta was related to the MAP2 contour distance as an indicator of dendritic segment density. To avoid bias, all quantifications were performed using the same predefined journals (Mokin and Keifer, 2006). Kv4.2/4.3 puncta were defined as an area $>5$ pixels $\left(0.01 \mu \mathrm{m}^{2}\right)$ to exclude unspecific background signals.

\section{$\mathrm{Ca}^{2+}$ imaging}

For time-lapse confocal recordings, a Yokogawa CSU-22 spinning disc confocal system (BFi OPTiLAS) was used with an Olympus BX-51WI upright microscope and a RedShirt NeuroCCD-SMQ camera (Life Imaging Services). Excitation was provided at $488 \mathrm{~nm}$ by a Coherent Sapphire 488-50 Laser (Coherent). Using the Olympus LumPlan FI 60×, 0.9 numerical aperture water-immersion objective, the power under the objective was typically $5-20 \mu \mathrm{W}$. When using the same objective, the lateral pixel size was $0.4 \mu \mathrm{m}$, and the pinhole size of the Yokogawa CSU-22 spinning disc confocal corresponded to 1.18 Airy units at $520 \mathrm{~nm}$ emission wavelength. Full frames were recorded at $125 \mathrm{~Hz}$. Imaging was started 20-30 min after breakthrough. For spine stimulation, the stimulation pipette was placed within $10-30 \mu \mathrm{m}$ of the imaged spine. When searching for spines, there is a bias against small spines and synapses with a very low $p$. Silent synapses will not be detected with synaptic stimulation at $-75 \mathrm{mV}$ holding potential. Spines were only used for experiments when stable responses could be elicited from well isolated $\mathrm{Ca}^{2+}$ sources (see Fig. 4A). Experiments were discarded if there was a change in absolute baseline brightness $>20 \%$ or increase in $\tau>20 \%$.

\section{Analysis}

All imaging data displayed (unless otherwise noted) and used for quantitative analysis are averages of three to five sweeps. When synaptically stimulating single spines for $\mathrm{Ca}^{2+}$ imaging, synaptic failures were not included in the averages to exclude presynaptic effects. The criterion for defining transmission failures and successes as well as significant backpropagating AP responses was a peak amplitude two times larger than the $\mathrm{SD}$ of the baseline noise. See Figure $4 A$ for distinction between successes and failures on the single-trial level. The signal amplitudes were obtained from exponential fits of averaged traces starting at signal initiation. Ratios were formed as pairwise comparisons in individual cells.

For LTP measurements, only experiments were included that displayed stable recording conditions for longer than $15 \mathrm{~min}$ after LTP induction. LTP was quantified for statistical comparisons by normalizing and averaging EPSC amplitudes between 20 and $25 \mathrm{~min}$ after induction to $4 \mathrm{~min}$ of baseline. In the 4-AP LTP experiments, the analysis was performed on a time interval 15-20 min after induction. Displayed traces are averages of the sweeps corresponding to this time interval.

All data are displayed as means \pm SEM. To determine statistical significances, we used two-tailed unpaired Student's $t$ tests or paired $t$ tests as appropriate, a Mann-Whitney $U$ test in which data was not normally distributed (Shapiro-Wilk test), or a one-way ANOVA, followed by Tukey's multiple comparison test. Differences were called significant if $p<0.05$.

\section{Results}

\section{Attenuation of the bAP-mediated dendritic $\mathrm{Ca}^{2+}$ signal in the} sensory layer 1a

Pyramidal cells in layer 2 of the anterior piriform cortex were identified by their typical morphology and intrinsic electrical properties (an initial burst fired after suprathreshold somatic current injection; shown in Fig. 1A) (Suzuki and Bekkers, 2006). A spinning disc confocal with a fast CCD camera was used for $\mathrm{Ca}^{2+}$ imaging. Neurons were loaded with the high-affinity $\mathrm{Ca}^{2+}$ indicator OGB1 or the medium-affinity $\mathrm{Ca}^{2+}$ indicator OGB6F for $25 \mathrm{~min}$ via the patch pipette before recording. Somatic APs were evoked by suprathreshold current injection (1-3 nA for 2 

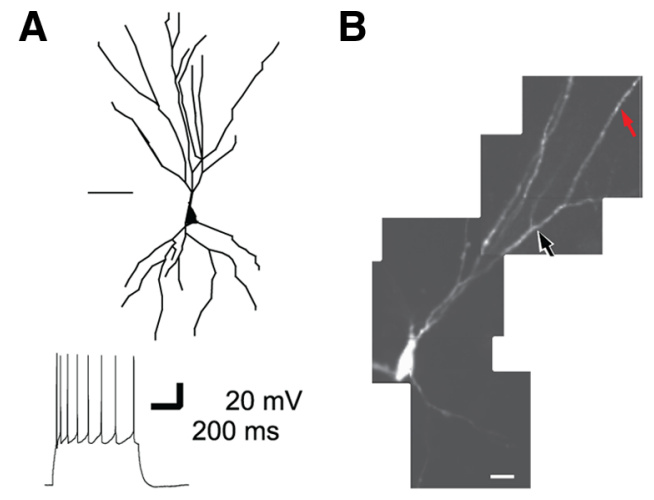

\section{OG-BAPTA1}

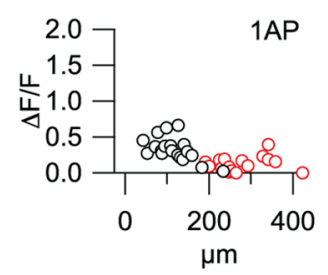

E OG-BAPTAGF

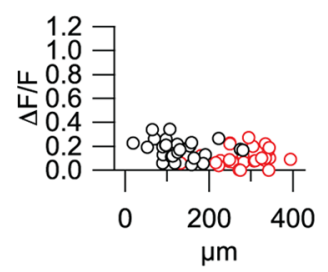

G

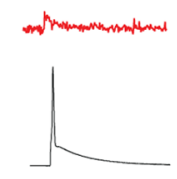

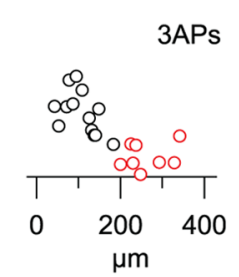

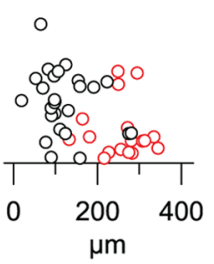

$50 \mathrm{~Hz}$

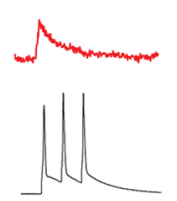

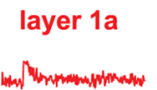
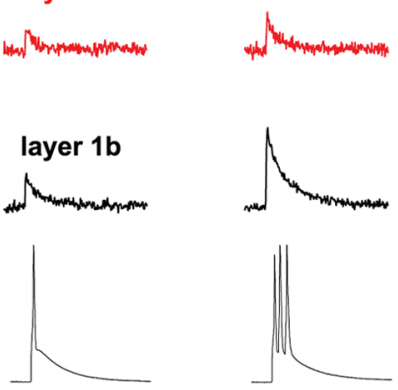

D
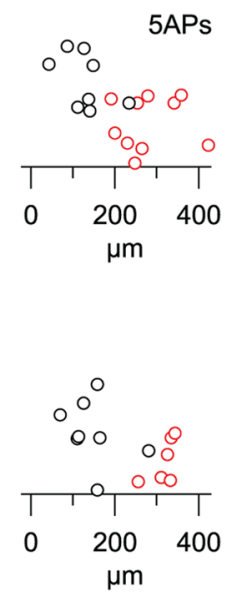

$100 \mathrm{~Hz}$

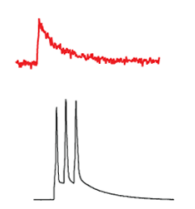

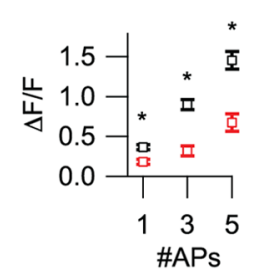

$\mathbf{F}$
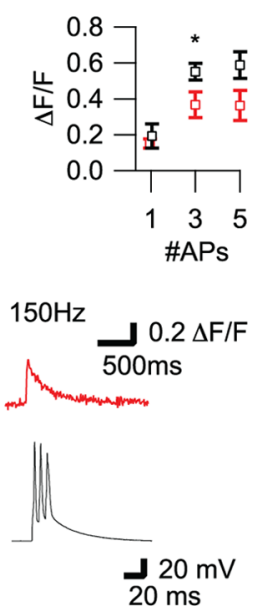
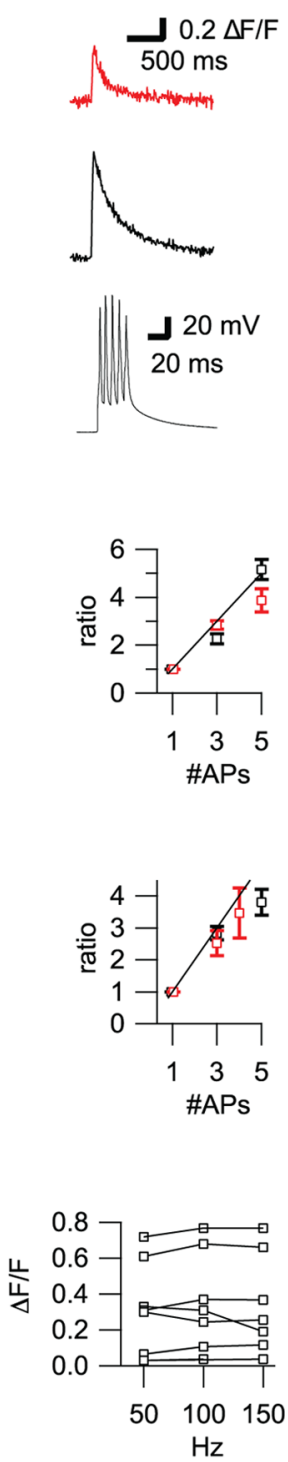

Figure 1. Properties of backpropagating AP-mediated dendritic $\mathrm{Ca}^{2+}$ signals in layer 1a and $1 \mathrm{~b}$. $\mathrm{A}$, Top, Camera lucida reconstruction of a biocytin-filled layer Il pyramidal cell in the anterior piriform cortex. Scale bar, $50 \mu \mathrm{m}$. Bottom, Firing pattern evoked by suprathreshold somatic current injection. B, Left, Montage of a layer 2 pyramidal cell in the anterior piriform cortex filled with OGB6F. Arrows point at dendritic regions corresponding to $\mathrm{Ca}^{2+}$ traces on the right. Right, $\mathrm{Ca}^{2+}$ signals in layer $1 \mathrm{a}$ (top) and layer $1 \mathrm{~b}$ (middle) evoked by one, three, and five $\mathrm{APs}$ at $150 \mathrm{~Hz}$ (bottom). C, Scatter plot of peak $\mathrm{Ca}^{2+}$ measured with OGB1 versus distance from the soma for one, three, and five APs. D, Left, Averaged peak amplitudes of significant responses are larger in layer $1 \mathrm{~b}$ than in layer 1a at one ( $n=17$ and 9 , respectively), three ( $n=13$ and 7, respectively), and five ( $n=10$ and 9, respectively; $\left.{ }^{*} p<0.01\right)$ APs. Right, Ratios of three (layer $1 \mathrm{~b}, n=12$; layer $\left.1 \mathrm{a}, n=5\right)$ and five (layer $1 \mathrm{~b}, n=9$; layer $1 \mathrm{a}, n=6$ ) APs over one AP display sublinear to linear addition of APs in layer 1a and layer $1 \mathrm{~b}$. $\boldsymbol{E}$, Scatter plot of peak $\mathrm{Ca}^{2+}$ measured with $0 \mathrm{~GB} 6 \mathrm{~F}$ versus distance from the soma for one, three, and five APs. $F$, Left, Averaged peak amplitudes of significant responses are larger in layer $1 \mathrm{~b}$ than in layer $1 \mathrm{a}$ at one $(n=23$ and 9, respectively; $p>0.1)$, three $(n=24$ and 11 , respectively; $\left.{ }^{*} p<0.05\right)$, and five ( $n=7$ and 4, respectively; $\left.p>0.05\right)$ APs. Right, Ratios of three (layer $1 \mathrm{~b}, n=18 ;$;ayer 1a, $\left.n=8\right)$ and five (layer $\left.1 \mathrm{~b}, n=7\right)$ or four (layer $1 \mathrm{a}, n=3$ ) APs over one AP display sublinear to linear addition of APs in layer 1 a and layer 1 b. G, Left, Time course of $\mathrm{Ca}^{2+}$ signals in layer 1a (top) evoked by one AP and three APs at 50,100 , and $150 \mathrm{~Hz}$ (bottom). Right, Plot of peak amplitudes of $\mathrm{Ca}^{2+}$ signals at 50,100 , and $150 \mathrm{~Hz}$.

ms). bAP-mediated $\mathrm{Ca}^{2+}$ responses were measured in different regions of the dendritic tree. The distance of the imaged dendritic region from the soma was measured using a post hoc reconstruction of the dye-filled cell (Fig. $1 B$ ). On small magnification differential interference contrast (DIC) images, layer la and layer $1 \mathrm{~b}$ could be distinguished by their different brightness (see Fig. 6A). In addition to the absolute distance, the synaptic layer was attributed to each imaging site by localizing corresponding image points on the DIC and the CCD image.

When using the high-affinity $\mathrm{Ca}^{2+}$ indicator OGB1, $\mathrm{Ca}^{2+}$ responses to single bAPs could be detected throughout the dendritic tree. There was a clear decrease in the amplitude of the
bAP-evoked $\mathrm{Ca}^{2+}$ response with distance from the soma (Fig. $1 C)$; the average amplitude of significant single bAP responses was $0.36 \pm 0.03 \Delta F / F(n=17)$ in layer $1 \mathrm{~b}$ and $0.18 \pm 0.03 \Delta F / F$ $(n=9)$ in layer 1a $(p<0.01)$.

\section{No regenerative $\mathrm{Ca}^{2+}$ responses to high-frequency bursts in distal dendrites}

In the apical dendrites of layer $2 / 3$ and layer 5 neocortical pyramidal cells and in basal dendrites of layer 5 cells, the distancedependent attenuation of the bAP-evoked $\mathrm{Ca}^{2+}$ response can be bypassed: short bursts of APs in the range of up to $150 \mathrm{~Hz}$ evoke regenerative supralinear dendritic $\mathrm{Ca}^{2+}$ responses in distal den- 
drites (Larkum et al., 1999a; Waters et al., 2003; Kampa and Stuart, 2006; Larkum et al., 2007). Applying comparable somatic high-frequency bursts, similar behavior of layer 2 pyramidal cells of the piriform cortex could not be confirmed. Based on observations from previous in vitro and in vivo experiments (Wilson, 1998; Suzuki and Bekkers, 2006), we tested bursts of three and five APs at $150 \mathrm{~Hz}$. For three APs, the average $\mathrm{Ca}^{2+}$ signal amplitude, as measured with OGB1, was $0.9 \pm 0.08$ $\Delta F / F$ in layer $1 \mathrm{~b}(n=13)$ and $0.32 \pm 0.06$ $\Delta F / F$ in layer $1 \mathrm{a}(n=7 ; p<0.01)$. The ratio of the $\mathrm{Ca}^{2+}$ response for three bAPs to that for one bAP was determined in individual cells. For layer $1 \mathrm{a}$ and $1 \mathrm{~b}$, ratio values were comparable $(2.84 \pm 0.18, n=$ 12 for layer $1 \mathrm{~b}$ and $2.26 \pm 0.41, n=5$ for layer 1a). Similar results were obtained with five AP bursts (Fig. 1D), suggesting that, at high $\left[\mathrm{Ca}^{2+}\right]$, the high-affinity $\mathrm{Ca}^{2+}$ indicator OGB1 might be saturated. For this reason, the experiments were repeated with the medium-affinity $\mathrm{Ca}^{2+}$ indicator OGB6F (Fig. 1E). In comparing effects of three bAPs and one bAP, the ratios of significant $\mathrm{Ca}^{2+}$ responses were $2.84 \pm 0.21(n=18)$ for layer $1 \mathrm{~b}$ and $2.52 \pm 0.39(n=8)$ for layer la (Fig. $1 F)$. This lack of supralinearity was also confirmed for the relationship between values associated with five and one bAPs in layer $1 \mathrm{~b}$ and four and one bAPs in layer $1 \mathrm{a}$. The observed absence of supralinearity in distal dendrites was consistent over a broad range of frequencies, again using the medium-affinity $\mathrm{Ca}^{2+}$ indicator OGB6F (Fig. 1G). The ratio of significant $\mathrm{Ca}^{2+}$ transients evoked by bursts of three APs at $150 \mathrm{~Hz}$ to that at $100 \mathrm{~Hz}$ was $1.00 \pm$ $0.08(n=5)$, and the ratio for values at 150 $\mathrm{Hz}$ to those at $50 \mathrm{~Hz}$ was $1.19 \pm 0.19(n=5)$.

\section{Synapse-specific dendritic compartmentalization mediated by A-type $\mathrm{K}^{+}$conductances}

The differential distribution of intrinsic conductances in layer $1 \mathrm{a}$ and layer $1 \mathrm{~b}$ could explain the observed attenuation of $\mathrm{Ca}^{2+}$ transients during bAP-induced voltage changes. For this reason, we examined the immunohistochemical distribution of $\mathrm{Kv} 4.2$ and $\mathrm{Kv} 4.3$, the two main subtypes constituting the dendritic A-type $\mathrm{K}^{+}$current (for review, see Birnbaum et al., 2004). Figure $2 A$ shows the higher expression levels of Kv4.2 and Kv4.3 in the distal dendrites. As seen at a high magnification, the surface expression of the $\mathrm{K}^{+}$ channel subunits in relation to the cytosolic MAP2 stain is higher in layer $1 \mathrm{a}$ than in layer $1 \mathrm{~b}$. The results are representative of five independently processed animals. As a result of neuronal branching, it is conceivable that the higher distal expression is based on a higher density of thinner dendritic branches in layer 1a. To address this question, we quantified the data morphometrically,
A
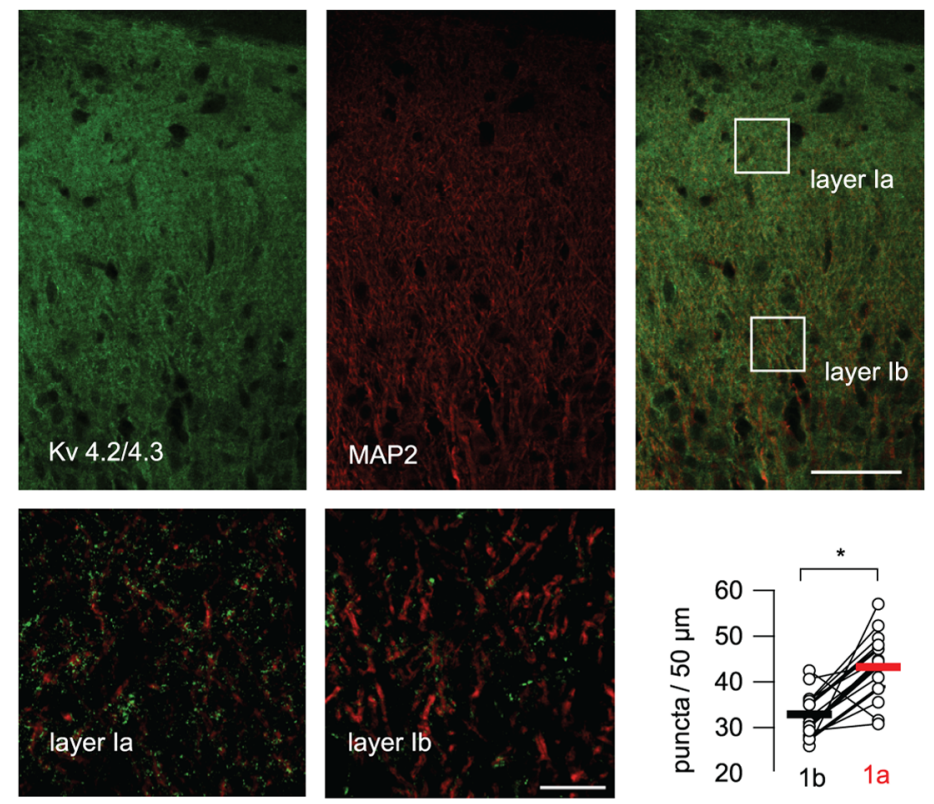

B layer $1 \mathrm{~b}$

layer 1a

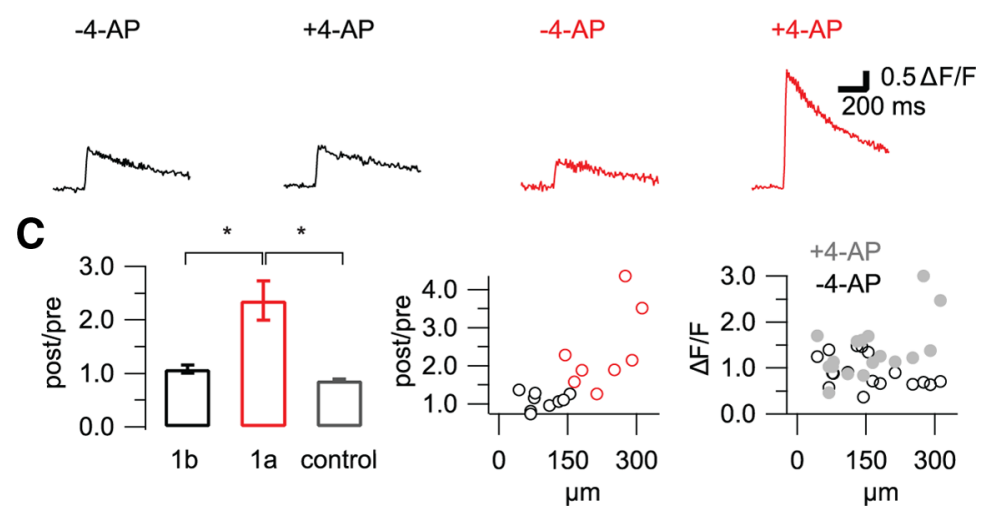

Figure 2. A distance-dependent increase in A-type $\mathrm{K}^{+}$conductances results in attenuation of distal $\mathrm{Ca}^{2+}$ signals in dendrites. A, Immunohistochemistry using MAP2- and Kv4.2/4.3-specific antibodies revealed different expression levels of A-type $\mathrm{K}^{+}$channels within the apical dendrites of the pyramidal cell. Top, Overview of the anterior piriform cortex demonstrating higher expression of Kv4.2/4.3 in distal dendrites corresponding to layer 1a. Scale bar, $100 \mu \mathrm{m}$. Bottom, High-magnification MAP2 and Kv4.2/ 4.3 stainings corresponding to the insets in the top merged picture from layer 1a and layer $1 \mathrm{~b}$ demonstrate the higher surface expression levels of Kv4.2/4.3 in layer 1a. Scale bar, $10 \mu \mathrm{m}$. Bottom right, Plot of the number of Kv4.2/4.3-positive puncta per 50 $\mu \mathrm{m}$ of dendritic length in corresponding layer $1 \mathrm{a}$ and layer $1 \mathrm{~b}$ segments. The Kv4.2/4.3 density is significantly higher in layer 1a ( $n=15$ slices from 5 animals; ${ }^{*} p<0.01$ ). $\boldsymbol{B}$, Time courses of dendritic $\mathrm{Ca}^{2+}$ signals evoked by three APs at $150 \mathrm{~Hz}$ and measured with 0GB1 from two different cells in layer $1 \mathrm{~b}$ (left) and layer 1a (right). Fifteen minutes of wash-in of 4-AP increases the Ca ${ }^{2+}$ signal only in layer 1a. C, Left, 4-AP increases the $\mathrm{Ca}^{2+}$ signals evoked by three APs at $150 \mathrm{~Hz}$ in layer $1 \mathrm{a}(n=9)$ but not in layer $1 \mathrm{~b}\left(n=8 ;{ }^{*} p<0.01\right)$ and controls $\left(n=5 ;{ }^{*} p<0.01\right)$. Middle, Plot of the ratio of peak Ca ${ }^{2+}$ signals post-4-AP over pre-4-AP application versus distance. The post/pre ratio increases with distance from the soma. Right, Plot of peak $\mathrm{Ca}^{2+}$ signals in 4-AP versus distance does not display the distance-dependent attenuation observed in control conditions.

normalizing the number of green puncta in layer $1 \mathrm{a}$ and layer $1 \mathrm{~b}$ to the dendritic length (see Materials and Methods). In layer 1b, we counted an average of $33.1 \pm 1.2 \mathrm{Kv} 4.2 / 4.3$ puncta per $50 \mu \mathrm{m}$ dendritic length. In the corresponding layer la segments, the Kv4.2/4.3 expression density was significantly increased to $43.4 \pm$ 1.9 puncta per $50 \mu \mathrm{m}$ dendritic length. This resulted in a layer 1a to $1 \mathrm{~b}$ expression ratio of $1.34 \pm 0.08(n=15$ slices from 5 animals; $p<0.01)$. To validate this result, we subsequently examined the effect of the A-type $\mathrm{K}^{+}$channel blocker 4-AP. Application of $4 \mathrm{~mm} 4$-AP resulted in a $2.4 \pm 0.37$-fold increase in the amplitude of the $\mathrm{Ca}^{2+}$ response evoked by three bAPs com- 
A

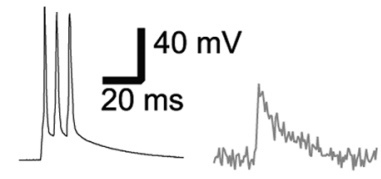

B OG-BAPTA1

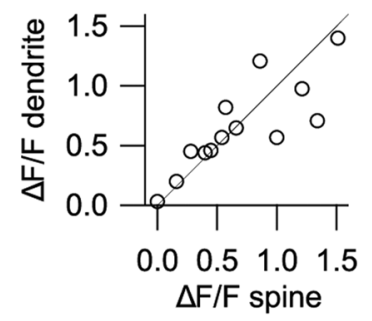

C

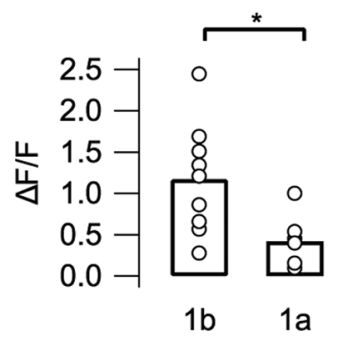

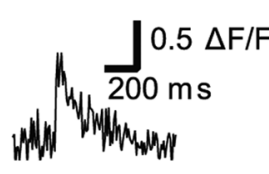

OG-BAPTA6F
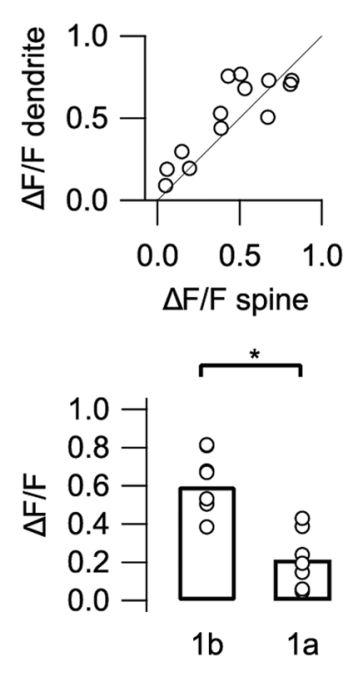

Figure 3. On the single-spine level, backpropagating AP-mediated $\mathrm{Ca}^{2+}$ signals also display attenuation in layer 1a. $\boldsymbol{A}$, Left, Time course of the $\mathrm{Ca}^{2+}$ signal in spine and dendrite evoked by three APs at $150 \mathrm{~Hz}$. Right, Dendritic branch with analyzed spine and dendrite indicated by black and gray arrows, respectively. Scale bar, $5 \mu \mathrm{m}$. B, Plot of peak $\mathrm{Ca}^{2+}$ signals in dendrites versus corresponding spines evoked by firing of three APs at $150 \mathrm{~Hz}$ with $0 G B 1$ (left) and 0GB6F (right). Spines display peak $\mathrm{Ca}^{2+}$ signals comparable with dendrites. $\boldsymbol{C}$, The average peak $\mathrm{Ca}^{2+}$ signal evoked by three APs at $150 \mathrm{~Hz}$ is significantly larger in layer $1 \mathrm{~b}$ spines than in layer $1 \mathrm{a}$ spines both with $0 \mathrm{~GB} 1$ ( $n=9$ and 6 , respectively; ${ }^{*} p<0.05$; left) and $0 \mathrm{~GB} 6 \mathrm{~F}$ ( $n=8$ and 7 , respectively; ${ }^{*} p<0.01$; right).

pared with preapplication values $(n=8)$ in individual cells in layer $1 \mathrm{a}$. In layer $1 \mathrm{~b}$, the same 4 -AP application did not change the amplitude of the $\mathrm{Ca}^{2+}$ response (post/pre $=1.08 \pm 0.07, n=9$; $p<0.01$ layer la vs layer 1b) (Fig. 2C). Drug-free control experiments yielded post/pre ratios of $0.87 \pm 0.02[n=5$ (Fig. $2 B) ; p<$ 0.01 layer 1a drug vs layer 1a control (Fig. $2 C$ )]. The smaller post value indicates a slight rundown of the dendritic $\mathrm{Ca}^{2+}$ response during the 15 min waiting period between the two measurements. To investigate the contribution of D-type $\mathrm{K}^{+}$channels, which are also affected by high 4-AP concentrations, we tested low concentrations of 4-AP (100 $\mu \mathrm{M})$ (Kampa and Stuart, 2006). At this 4 -AP concentration, affecting primarily $\mathrm{D}$-type $\mathrm{K}^{+}$channels, the post/pre ratios were $1.37 \pm 0.24$ and not significantly different from the drug-free control experiments $(n=5 ; p>$ 0.05 , layer 1 a drug vs layer 1 a control). We conclude that the differential distribution of A-type $\mathrm{K}^{+}$channels in the apical dendrite of layer 2 pyramidal cells of the piriform cortex, i.e., higher expression levels in layer $1 \mathrm{a}$ than layer $1 \mathrm{~b}$, results in the functional compartmentalization of the dendrites into two segments.

\section{Different spine $\mathrm{Ca}^{2+}$ signals mediated by action potential backpropagation in layer $1 \mathrm{a}$ and layer $1 \mathrm{~b}$}

Dendritic spines constitute the subcellular compartment in which the actual synaptic contacts that define the input layers are located. The differences between distinct synaptic layers with respect to dendritic $\mathrm{Ca}^{2+}$ responses to bAPs were analyzed at

the single-spine level. We initially compared the amplitude of bAP-evoked $\mathrm{Ca}^{2+}$ transients in spines and dendrites (Fig. $3 A, B)$. We observed no significant differences between the bAPmediated $\mathrm{Ca}^{2+}$ responses of the two subcellular compartments. Using OGB1, the ratio of the response amplitude between dendrite and spine was $1.03 \pm 0.1(n=12)$. With OGB6F, this dendrite/spine ratio was $1.44 \pm 0.18(n=13)$. The trend that somewhat larger dendritic $\mathrm{Ca}^{2+}$ transients were observed with the medium-affinity indicator was not statistically significant $(p>0.05)$, and it can be explained by the reduced degree of dendrite-spine diffusion of indicator-bound $\mathrm{Ca}^{2+}$ attributable to the lower buffering capacity (Sabatini et al., 2002) (for review, see Higley and Sabatini, 2008). As seen for dendritic transients, the amplitudes of bAP-mediated $\mathrm{Ca}^{2+}$ responses were different for spines in the two input layers, with significantly larger responses observed in layer $1 \mathrm{~b}$ using both OGB1 and OGB6F (Fig. 3C).

\section{Spine $\mathrm{Ca}^{2+}$ transients mediated by synaptic stimulation in layer $1 \mathrm{a}$ and layer $1 \mathrm{~b}$}

The next step was to measure $\mathrm{Ca}^{2+}$ transients in dendritic spines evoked by subthreshold synaptic stimulation. EPSPs are expected to result in specific and large $\mathrm{Ca}^{2+}$ transients in spines relative to dendrites (Yuste and Denk, 1995). Synaptic stimulation resulted in isolated spine $\mathrm{Ca}^{2+}$ transients, with dendrite/spine ratios of $0.29 \pm 0.07(n=9)$ for experiments with OGB1 and $0.32 \pm 0.07(n=13)$ when OGB6F was used (Fig. $4 A, C)$. These values are in accordance with previously published ratios measured using OGB1 and two-photon imaging (Nevian and Sakmann, 2004). As shown in Figure 4A, synaptic stimulation was synapse specific. The overlay of several single responses (Fig. $4 A$, bottom) reveals that both successful synaptic transmission and synaptic failures could be detected. This mirrors the probabilistic nature of synaptic transmission at the spine $\mathrm{Ca}^{2+}$ level (Yuste and Denk, 1995). Spine $\mathrm{Ca}^{2+}$ transients evoked by synaptic stimulation were almost completely blocked after a 5 min application of the NMDAR antagonist D-APV at $50 \mu \mathrm{M}$ (reduction to $11 \pm 3 \%$ of preapplication values, $n=5 ; p<0.01$ ) (Fig. $4 B$ ). When comparing the amplitudes of synaptically activated spine $\mathrm{Ca}^{2+}$ transients in layer $1 \mathrm{a}$ and layer $1 \mathrm{~b}$, there was no significant difference observed for either of the $\mathrm{Ca}^{2+}$ indicators (OGB1: layer 1b, $0.99 \pm 0.14 \Delta F / F, n=7$; layer $1 \mathrm{a}, 0.83 \pm 0.12$ $\Delta F / F, n=4 ; p>0.05$; OGB6F: layer 1b, $0.6 \pm 0.09 \Delta F / F, n=6$; layer la, $0.48 \pm 0.07 \Delta F / F, n=8 ; p>0.05$ ) (Fig. $4 D$ ).

We observed a large variability of the somatic voltage recorded with the imaged spines (Fig. $4 E$ ), based on the variable number and strength of the synapses activated with extracellular stimulation. In most CNS synapses, a single axon constitutes several diffusely distributed synaptic contacts to a given postsynaptic cell (Shepherd, 2004). The anatomical data characterizing the number of synaptic contacts per pair of connected cells is missing for the piriform cortex. Regardless of the underlying axonal projection patterns, the primary relevant concern here is that the coincident activation of several neighboring spines might result in a dendritic depolarization. This would cause additional spine $\mathrm{Ca}^{2+}$ influx and result in distortion of the EPSP-mediated signal. APmediated $\mathrm{Ca}^{2+}$ signals that result in global dendritic depolarization are as large as or larger than the associated spine responses (Fig. 4C). In contrast, the observed EPSP-mediated signals are larger in the spine than in the dendrite (Fig. 4D). This argues against significant dendritic depolarization. In addition, the fullframe scan applied with the spinning disc confocal permits for 
A

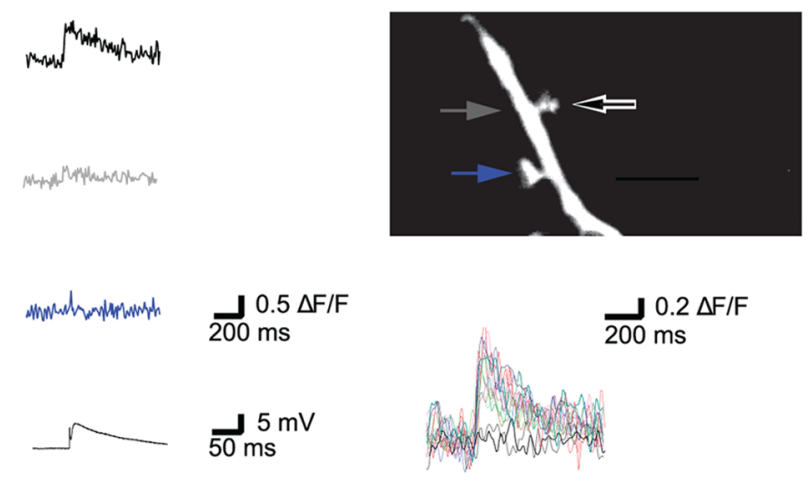

B

-APV
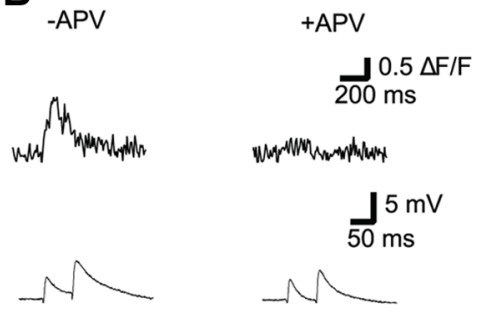
$-N$
C OG-BAPTA1

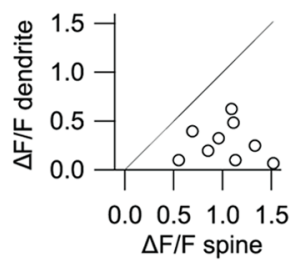

D

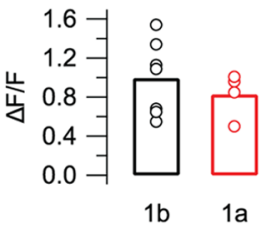

$\mathbf{E}$
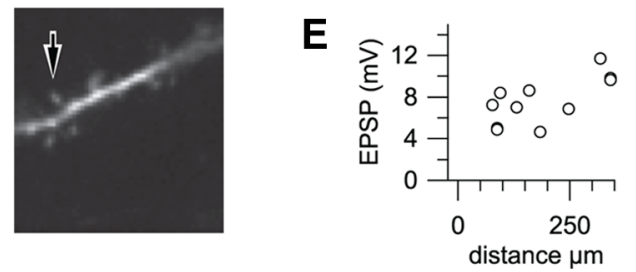

OG-BAPTA6F
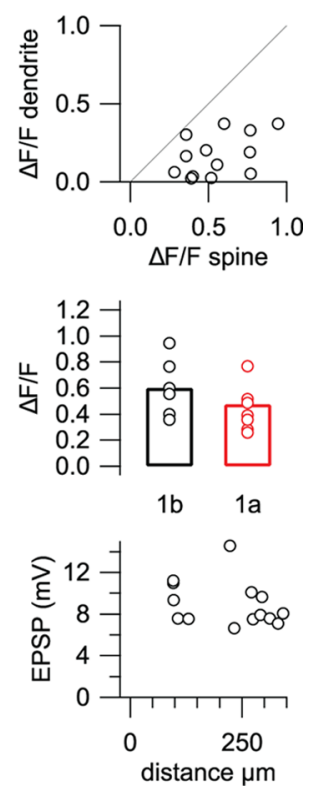

Figure 4. Single-spine $\mathrm{Ca}^{2+}$ signals evoked by synaptic stimulation are NMDAR dependent and do not differ between layer 1a and layer 1b. $A$, Left, Time course of EPSP-mediated Ca ${ }^{2+}$ signal in stimulated spine, adjacent dendrite, and unstimulated spine. Top right, Dendritic branch with active spine (black arrow), adjacent dendrite (gray arrow), and inactive spine (blue arrow). This image was acquired with a high-resolution $C C D$ camera different from the $C C D$ camera used for detecting $\mathrm{Ca}^{2+}$ signals. Scale bar, $5 \mu \mathrm{m}$. Bottom right, The overlay displays Gaussian filtered time courses of single EPSP-mediated $\mathrm{Ca}^{2+}$ signals from the active spine and demonstrates distinguishable synaptic failures. $\boldsymbol{B}$, Left, Time course of EPSP-mediated $\mathrm{Ca}^{2+}$ transient in stimulated spine evoked by paired-pulse stimulation at $20 \mathrm{~Hz}$ before D-APV application. Middle, Time course of EPSP-mediated Ca ${ }^{2+}$ signal in the same spine after 5 min of $50 \mu \mathrm{m} \mathrm{D}-\mathrm{APV}$ reveals almost complete block of the spine $\mathrm{Ca}^{2+}$ transient. Right, Dendritic branch with analyzed spine (black arrow). Scale bar, $5 \mu \mathrm{m}$. C, Plot of peak $\mathrm{Ca}^{2+}$ signals in dendrites versus corresponding spines evoked by synaptic stimulation measured with $0 G B 1$ (left) and $0 G B 6 F$ (right). During synaptic stimulation, spines exhibit larger peak $\mathrm{Ca}^{2+}$ signals than dendrites. $\boldsymbol{D}$, The average peak $\mathrm{Ca}^{2+}$ signal in spines evoked by synaptic stimulation is comparable in layer $1 \mathrm{~b}$ and layer $1 \mathrm{a}$ for $0 \mathrm{GB1}$ ( $n=7$ and 4 , respectively; $p>0.05$; left) and $0 \mathrm{~GB} 6 \mathrm{~F}$ ( $n=6$ and 8 , respectively; $p>0.05$; right). $\boldsymbol{E}$, Plot of EPSP amplitude versus distance of the corresponding imaged spine from the soma. Left, Spines recorded with OGB1. Right, Spines recorded with OGB6F.

scanning of a larger dendritic segment. Significant dendritic voltage changes were further excluded by the absence of voltage change-mediated $\mathrm{Ca}^{2+}$ transients in dendritic segments adjacent to unstimulated spines.

\section{Calcium transients measured during subsequent presynaptic activation and postsynaptic bursting reveal different} integrative properties of layer $1 \mathrm{a}$ and layer $1 \mathrm{~b}$ spines

We demonstrated that, in layer 2 pyramidal cells of the piriform cortex, the spine $\mathrm{Ca}^{2+}$ influx during synaptic activation is mediated by NMDARs (Fig. $4 B$ ). LTP can be induced in pyramidal cells when the presynaptic firing is followed by postsynaptic spiking at a time interval of up to $50 \mathrm{~ms}$ (Magee and Johnston, 1997; Markram et al., 1997; Bi and Poo, 1998; Debanne et al., 1998) (for review, see Kampa et al., 2007). During LTP induction, bAPs can boost the spine $\mathrm{Ca}^{2+}$ response by depolarization-mediated release of the NMDAR $\mathrm{Mg}^{2+}$ block (Koester and Sakmann, 1998; Kampa et al., 2004; Nevian and Sakmann, 2004, 2006). The integration of presynaptic and postsynaptic firing in spines in the layer 1a and layer 1b dendritic compartments was analyzed. During presynaptic and postsynaptic stimulation at a time interval of $10 \mathrm{~ms}$, we computed the average amplitude of the spine $\mathrm{Ca}^{2+}$ transient. Under these conditions, the high-affinity indicator OGB1 was already partially saturated, so the medium-affinity indicator OGB6F was used. The amplitude in layer $1 \mathrm{~b}$ exceeded that of layer 1a by almost twofold (layer 1b, $1.62 \pm 0.38 \Delta F / F, n=$ 6 vs layer 1a, $0.84 \pm 0.12 \Delta F / F, n=7 ; p<0.01$ ) (Fig. $5 A-C$ ). Supralinear summation means that coincident synaptic stimula- tion and postsynaptic firing lead to a larger $\mathrm{Ca}^{2+}$ response than expected from the linear sum of the isolated responses (Fig. $5 A, B)$. Associative layer $1 \mathrm{~b}$ spines exhibit a significantly larger degree of supralinearity when compared with sensory layer $1 \mathrm{a}$ spines (layer 1b, $1.42 \pm 0.05, n=6$ vs layer $1 \mathrm{a}, 1.15 \pm 0.01, n=$ $7 ; p<0.05$ ) (Fig. $5 A-C$ ). The voltage-dependent relief of the $\mathrm{Mg}^{2+}$ block of the NMDAR is the underlying mechanism for the supralinear summation of the spine $\mathrm{Ca}^{2+}$ response by coincident presynaptic and postsynaptic firing (Nevian and Sakmann, 2004). We wanted to ensure that the initial EPSP-mediated voltage changes did not have an impact on the linearity index. We therefore plotted the depolarization, mediated by the EPSP, against the linearity index measured in individual experiments, and no correlation was observed (Fig. 5C).

Spikes were delivered as $150 \mathrm{~Hz}$ triplets to increase the efficiency of action potential backpropagation to distal dendrites (for review, see Kampa et al., 2007). These postsynaptic bursts have been observed with sensory and associative inputs to layer $2 / 3$ pyramidal cells in the piriform cortex in vitro (Suzuki and Bekkers, 2006) and in vivo (Wilson, 1998). However, isolated layer 1a stimulation has been shown recently to recruit prominent perisomatic feedforward inhibition, and single APs are the predominant cellular output during suprathreshold extracellular stimulation in layer 1a (Luna and Schoppa, 2008). We asked how this tightly controlled output mode affects the integration of presynaptic and postsynaptic firing in layer $1 \mathrm{~b}$ spines. Pairing an EPSP with one or three bAPs, the amplitudes and linearity indices of spine $\mathrm{Ca}^{2+}$ responses were 
A layer $1 \mathrm{a}$

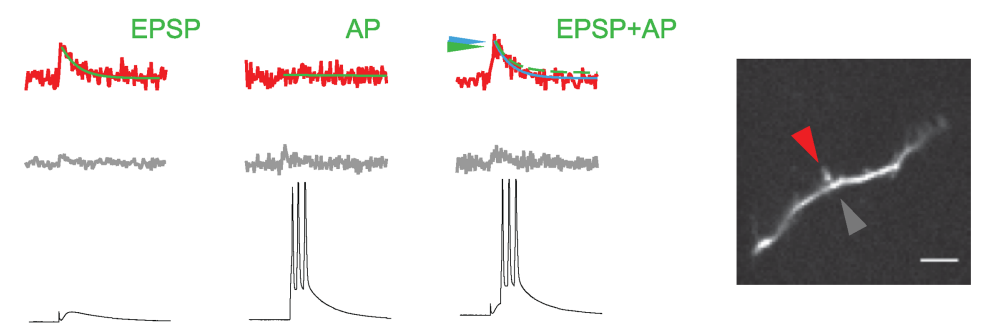

B layer $\mathbf{1 b}$
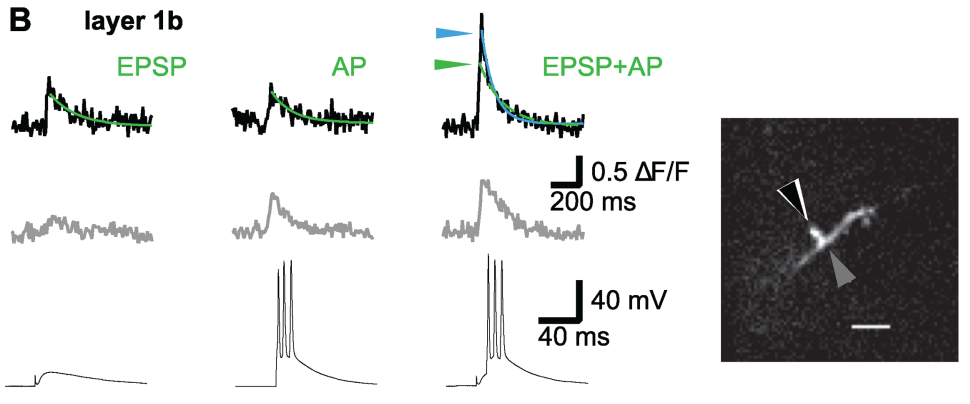

C
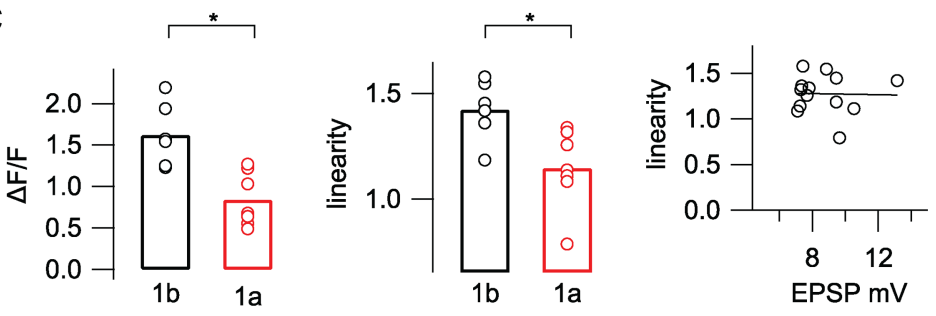

D

E
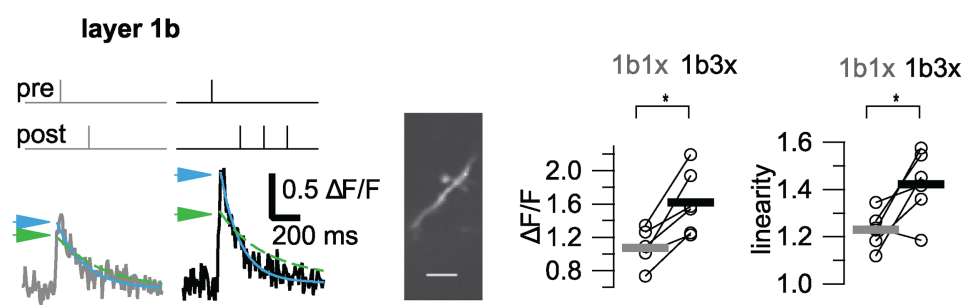

Figure 5. Pairing of EPSPs and APs results in supralinear increase of spine $\mathrm{Ca}^{2+}$ signals in layer $1 \mathrm{~b}$ only. $A$, Left, Comparison of $\mathrm{Ca}^{2+}$ signals in spines (top) and adjacent dendrites (middle) evoked by an EPSP, three APs at $150 \mathrm{~Hz}$, and a pairing sequence in which a EPSP is followed by three APs at $150 \mathrm{~Hz}$ with a $\Delta t$ of $10 \mathrm{~ms}$ (bottom) in a layer 1a spine. The green lines are monoexponential fits of the EPSP and AP-mediated spine $\mathrm{Ca}^{2+}$ signals, and the fit of the pairing sequence is represented by the blue line. The dotted green line in the trace of the pairing sequenceis the linear sum of the fits of the isolated EPSP and AP. The arrows point at the peaks of the two fits. Right, Dendritic branch with analyzed spine (red arrow) and adjacent dendriticsegment (gray arrow). Scale bar, $5 \mu \mathrm{m}$. This example displays almostlinear addition of the spine $\mathrm{Ca}^{2+}$ signal during pairing of the EPSP with the APS. B, Left, Comparison of $\mathrm{Ca}^{2+}$ signals in spines and adjacent dendrites evoked by EPSPs, three APs at $150 \mathrm{~Hz}$, and a pairing sequence consisting of an EPSP followed by three APs at $150 \mathrm{~Hz}$ with a $\Delta t$ of $10 \mathrm{~ms}$ in a layer $1 \mathrm{~b}$ spine. The color code of the monoexponential fits is identical to $A$. Right, Dendritic branch with analyzed spine (black arrow) and adjacent dendritic segment (gray arrow). Scale bar, $5 \mu \mathrm{m}$. This example displays supralinear addition of the spine $\mathrm{Ca}^{2+}$ signal during pairing of the EPSP with the APs. C, Left, When pairing an EPSP with three APs at $150 \mathrm{~Hz}$ at a $\Delta t$ of $10 \mathrm{~ms}$, the averaged amplitudes of the $\mathrm{Ca}^{2+}$ signals in layer $1 \mathrm{~b}$ spines $(n=6)$ are significantly larger than in layer 1a spines $\left(n=7 ;{ }^{*} p<0.01\right)$. Middle, Layer $1 \mathrm{~b}$ spines $(n=6)$ display a significantly larger supralinear addition of EPSPs and APs when compared with layer 1a spines $(n=7 ; p<0.05)$. Right, Plot of the linearity indices measured in spines versus the corresponding EPSP amplitudes. The linearity indices in spines are independent of the EPSP amplitudes. D, Left, Time course of spine $\mathrm{Ca}^{2+}$ signals in layer $1 \mathrm{~b}$ spines during pairing of an EPSP with one AP (EPSP-AP sequence; see schematic illustration on top). The blue line represents the monoexponential fit of the paired EPSP-AP sequence, and the green line represents the linear sum of the fits of the isolated EPSP and AP. The arrows point at the peak amplitude of the fits. Middle, Time course of $\mathrm{Ca}^{2+}$ signals in layer $1 \mathrm{~b}$ spines during pairing of an EPSP with three APs at $150 \mathrm{~Hz}$ (EPSP-three AP sequence; see schematic illustration on top). The color code of the monoexponential fits is identical to the left. Comparison reveals a larger supralinear addition of the spine $\mathrm{Ca}^{2+}$ signal when the EPSP is followed by three APs instead of one AP. Right, Dendritic branch with analyzed spine. Scale bar, $5 \mu \mathrm{m}$. E, Left, Plot of the peak $\mathrm{Ca}^{2+}$ signals from spines stimulated with paired EPSP-AP and EPSP-three AP sequences in layer $1 \mathrm{~b}(n=6)$. The amplitude is significantly larger when the EPSP is paired with three APs $\left({ }^{*} p<0.05\right)$. Right, Plot of the linearity indices from spines stimulated with EPSP-AP and EPSP-three AP sequences in layer $1 \mathrm{~b}(n=6)$. The linearity indexis significantly larger when the EPSP is paired with three APs $\left({ }^{*} p<0.05\right)$. compared (Fig. 5D). The average spine $\mathrm{Ca}^{2+}$ signal increased from $1.07 \pm 0.09$ $\Delta F / F$ for one AP to $1.62 \pm 0.15 \Delta F / F$ for three APs $(n=6 ; p<0.05)$ (Fig. $5 E)$. The linearity index changed from an average value of $1.23 \pm 0.03$ for one AP to $1.42 \pm$ 0.05 for three APs $(n=6 ; p<0.05)$ (Fig. 5E).

\section{Pathway-specific LTP induction}

In pyramidal cells in CA1 and the neocortex, the supralinear spine $\mathrm{Ca}^{2+}$ responses are considered to be the elementary building blocks of LTP induction, with paradigms based on pairing EPSPs with subsequent bursts of APs (Pike et al., 1999; Kampa et al., 2006; Letzkus et al., 2006; Nevian and Sakmann, 2006). We wanted to relate the observed differences between spine $\mathrm{Ca}^{2+}$ responses in layer 1a and those in layer $1 \mathrm{~b}$ to the induction of LTP. For this purpose, LTP was induced in both layers using a stimulation protocol based on the stimuli displayed in Figure 5. To ensure pathway specificity of our LTP measurements, we first established the selective synaptic stimulation of layer 1a and layer $1 \mathrm{~b}$ synapses on apical dendrites of layer 2 pyramidal neurons. Electrodes were placed at the layer 2 /layer 1 b border for $1 \mathrm{~b}$ stimulation and at the LOT/ layer 1a border for 1a stimulation. It has been established previously that presynaptic $\mathrm{GABA}_{\mathrm{B}}$ receptors are exclusively expressed on layer $1 \mathrm{~b}$ synapses. It follows from this that application of the $\mathrm{GABA}_{\mathrm{B}}$ agonist baclofen selectively diminishes layer $1 \mathrm{~b}$ inputs, leaving layer 1a essentially unaffected (Tang and Hasselmo, 1994; Franks and Isaacson, 2005). In a subset of our LTP experiments, we confirmed the selective stimulation of the two different pathways by postexperimental application of $30 \mu \mathrm{M}$ baclofen. Between 3 and 4 min after baclofen application, we observed a reduction of layer $1 \mathrm{~b}$ inputs to $17.8 \pm 2.7 \%(n=7)$ of baseline values. The layer 1a inputs remained stable at $99 \pm 9.3 \%(n=6)$ of baseline level $(p<$ 0.01 ) (Fig. 6B). Selective stimulation of layer 1a by pharmacological block of layer 1 b synapses with baclofen has been demonstrated previously (Franks and Isaacson, 2005; Poo and Isaacson, 2007); however, it is important to note that $\mathrm{GABA}_{\mathrm{B}}$ receptors activate dendritic $\mathrm{K}^{+}$ conductances and inhibit voltage-gated calcium channels (VGCCs) in the postsynaptic cell (Pérez-Garci et al., 2006). The pharmacological isolation approach thus activates mechanisms that diminish layer $1 \mathrm{a} \mathrm{Ca}^{2+}$ responses evoked by bAPs and is therefore unsuited for our 
A

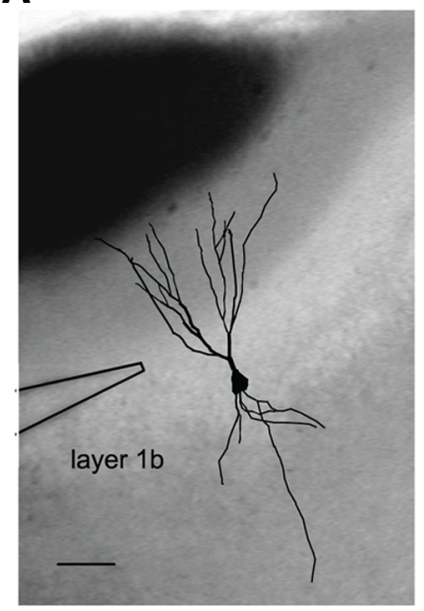

C
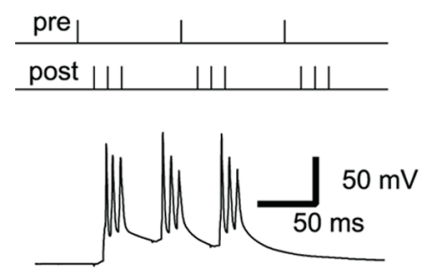

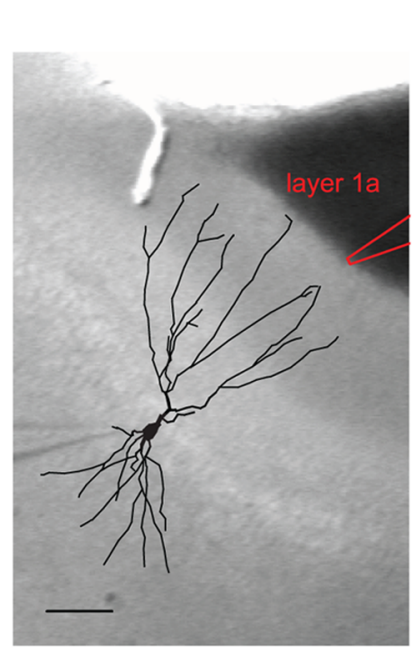

D

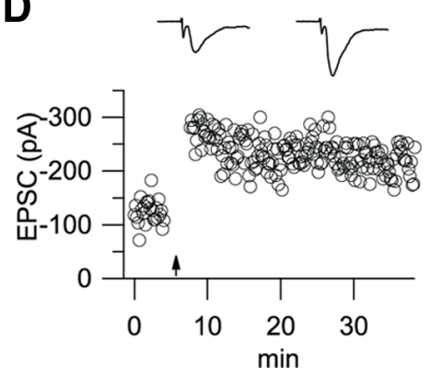

B
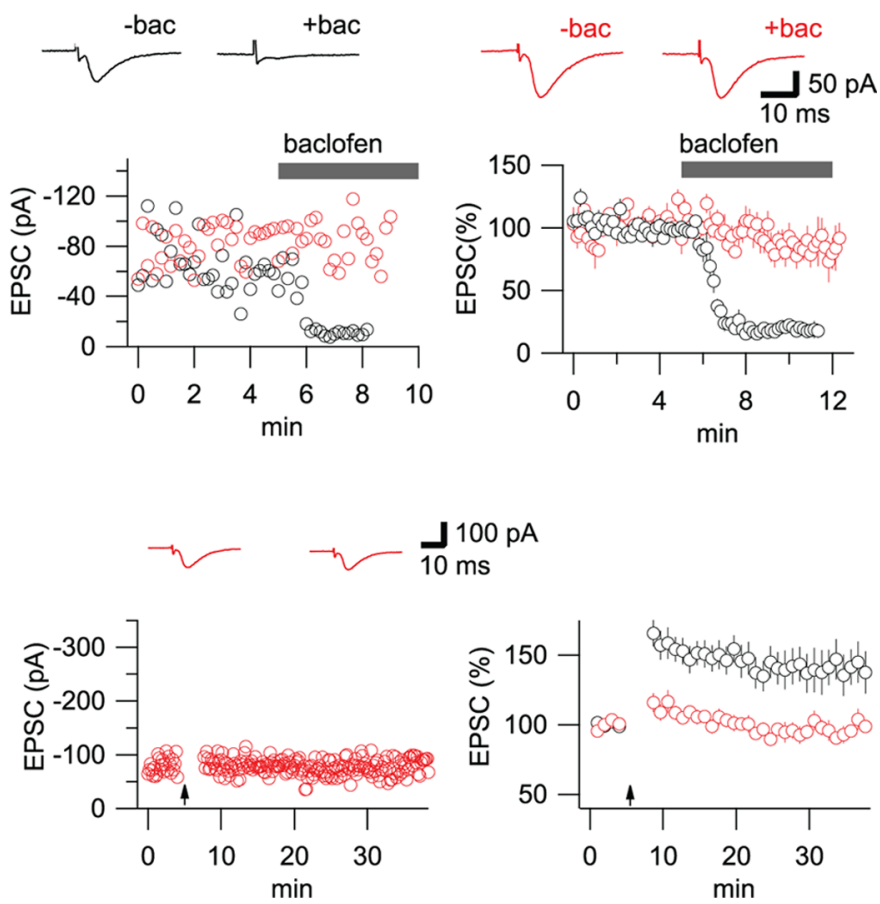

Figure 6. Pairing EPSPs with postsynaptic AP bursts only induces LTP in layer 1b. A, Left, Camera lucida reconstruction of recorded layer 2 pyramidal cell in the anterior piriform cortex projected onto a DIC image of the acute brain slice. The stimulation electrode positioned in layer $1 \mathrm{~b}$ is highlighted in black. Right, Camera lucida reconstruction of recorded layer 2 pyramidal cell in the anterior piriform cortex projected onto a DIC image of the acute brain slice. The stimulation electrode positioned in layer 1a is highlighted in red. Scale bars correspond to $100 \mu \mathrm{m}$. B, Top left, Corresponding evoked EPSCs before and 3 min after baclofen (bac) application from the cell in the left panel of $\boldsymbol{A}$ demonstrates block of evoked EPSCs by baclofen. Top right, Evoked EPSC before and 3 min after baclofen application from the cell in the $A$. Compared with the layer $1 \mathrm{~b}$ stimulation in the left, the EPSC evoked by layer 1a stimulation is unaffected by blocking GABA ${ }_{B}$ receptors. Bottom left, Time course of evoked EPSC amplitudes for the cells in the left panel of $\boldsymbol{A}$ (black) and the right panel of $\boldsymbol{A}$ (red), demonstrating the exclusive effect of baclofen on layer 1b. Bottom right, Time course of normalized amplitudes of layer $1 \mathrm{a}(n=6)$ and layer $1 \mathrm{~b}(n=7)$ inputs in response to baclofen application. GABA receptor block via baclofen results in selective reduction of layer $1 \mathrm{~b} \operatorname{EPSCS}(p<0.01)$. C, Top, Schematic illustration of the LTP induction protocol consisting of 30 bursts of EPSP triplets at $20 \mathrm{~Hz}$ followed by a $150 \mathrm{~Hz}$ triple AP burst $8 \mathrm{~ms}$ after each EPSP. Bottom, Voltage trace of a typical induction stimulus. D, Bottom left, Time course of EPSC amplitude changes induced by the LTP induction protocol (arrow) in a layer $1 \mathrm{~b}$ input. Top left, Corresponding averaged EPSCs 4 min before and $20-25$ min after LTP induction in layer $1 \mathrm{~b}$ (black). Bottom middle, Time course of EPSC amplitude changes induced by the LTP induction protocol (arrow) in a layer 1a input. Top middle, Corresponding averaged EPSCS 4 min before and $20-25$ min after LTP induction in layer 1a (red). Right, Time course of normalized EPSC amplitude changes induced by the LTP induction protocol (arrow) in layer 1a $(n=7$; red) and layer 1b $(n=10$; black) demonstrating the significantly larger potentiation in layer $1 \mathrm{~b}(p<0.05)$.

study. The observed selective reduction of layer $1 \mathrm{~b}$ inputs during baclofen application demonstrates that pathway-specific stimulation can be achieved with stimulation electrode placement alone (Fig. 6A, B).

LTP was induced by presynaptic stimulation that resulted in EPSPs paired with subsequent postsynaptic triple AP bursts at a time interval of $8 \mathrm{~ms}$. The EPSP-three AP sequences were delivered 30 times every $5 \mathrm{~s}$ in $20 \mathrm{~Hz}$ triplets (Fig. 6C). The $20 \mathrm{~Hz}$ input pattern matches the beta oscillations reported for odor-evoked synaptic currents in the piriform cortex (Poo and Isaacson, 2009). Using the paradigm displayed in Figure $6 C$ on both layer $1 \mathrm{~b}$ and layer 1a inputs, we successfully induced LTP in layer $1 \mathrm{~b}$ but failed to induce LTP in layer 1a (Fig. 6D). Between 20 and 25 min after LTP induction, cells stimulated in layer $1 \mathrm{~b}$ were potentiated at an average value of $138.6 \pm 13 \%(n=10)$ of preinduction baseline. This was significantly larger than that after layer 1a stimulation, in which cells did not show any potentiation: the EPSC amplitude was $97 \pm 6 \%(n=7)$ of baseline values $(p<0.05)$.

\section{Rescue of distal layer 1a LTP by enhanced AP backpropagation}

We next analyzed the effect of enhanced AP backpropagation and the resulting change in intracellular $\mathrm{Ca}^{2+}$ on the induction of distal LTP. Blocking A-type $\mathrm{K}^{+}$conductances with $4 \mathrm{~mm}$ 4-AP substantially reduces the weak distal depolarization during bAP firing and enhances distal voltage-mediated $\mathrm{Ca}^{2+}$ influx (Fig. 2). Using our EPSP-triple AP-based LTP induction paradigm in the presence of 4-AP, distal LTP could be evoked. Between 15 and $20 \mathrm{~min}$ after LTP induction, layer $1 \mathrm{a}$ inputs were potentiated at an average of $143 \pm 18 \%(n=8)$ of preinduction baseline values (Fig. $7 A, C$ ). In contrast, with the EPSP-based induction protocol in 4-AP, and also with the EPSP-triple AP-based layer 1a LTP induction protocol without 4-AP, cells exhibited no potentiation. The EPSC amplitude was $68 \pm 10 \%$ $(n=7)$ and $95 \pm 13 \%(n=8)$ of baseline values, respectively $(p<0.01$ and $p<0.05)$ (Fig. 7C). This demonstrates the instructive role of bAP-associated distal dendritic depolarization and the associated increase in the distal $\mathrm{Ca}^{2+}$ response for LTP in layer $1 \mathrm{a}$.

In layer $\mathbf{1 b}$, bursting is a prerequisite for plasticity induction We examined the induction of LTP in different layers using postsynaptic AP triplets. As described previously, we compared the effect of different output modes on the integration of presynaptic and postsynaptic firing in layer $1 \mathrm{~b}$ spines with respect to the associated spine $\mathrm{Ca}^{2+}$ responses (Fig. 5D). We subsequently de- 

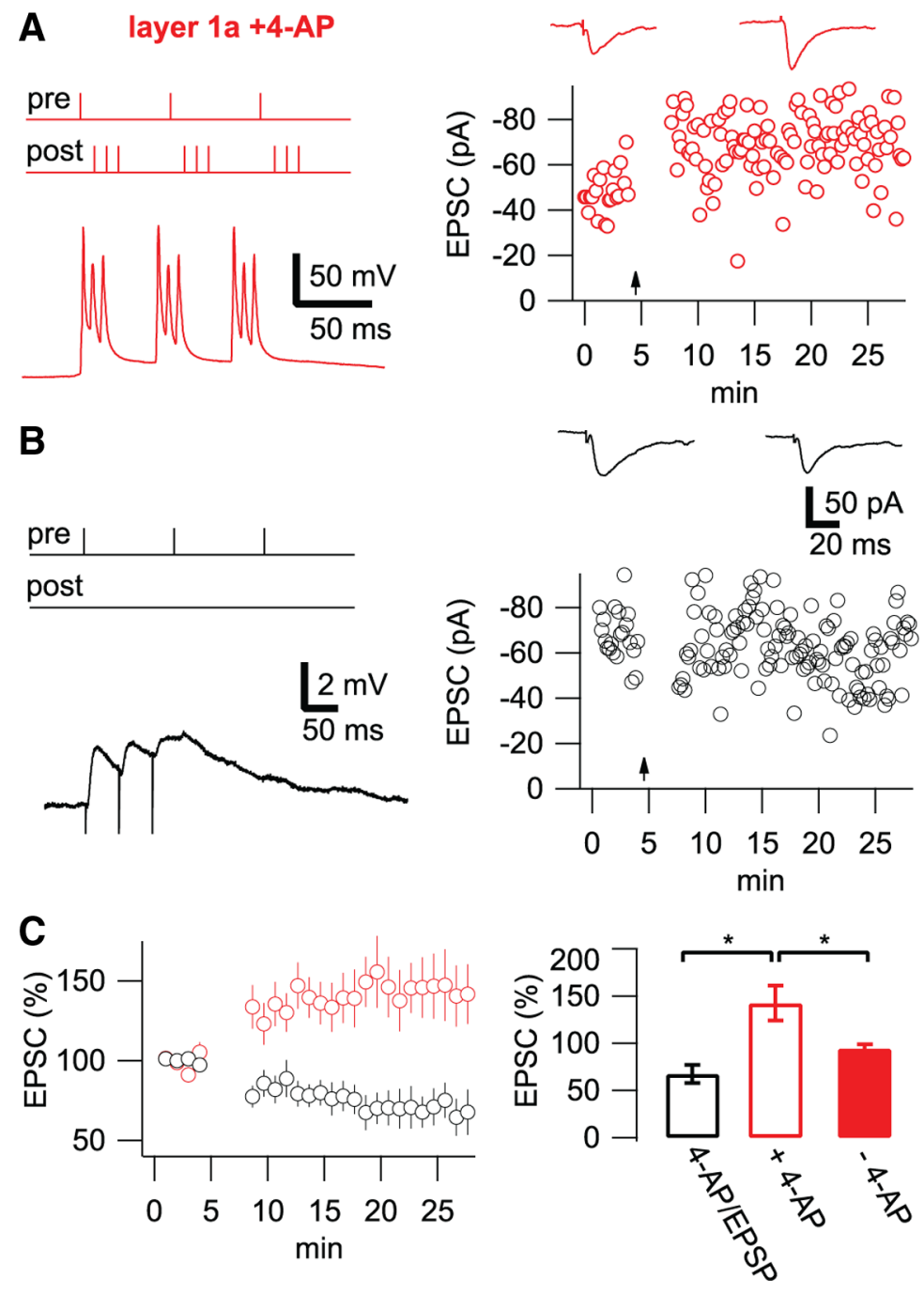

Figure 7. LTP in layer 1a can be induced when bAPs are amplified in layer 1a using 4-AP.A, Left, Schematic illustration of the LTP induction protocol in 4-AP and voltage trace of a typical induction stimulus (top). Right bottom, Time course of EPSC amplitude changes induced by the LTP induction protocol (arrow) in a layer 1a input in 4-AP. Right top, Corresponding averaged EPSCs 4 min before and 15-20 min after LTP induction in layer 1a. B, Left, Schematic illustration of the control protocol in 4-AP consisting of 30 bursts of EPSP triplets at $20 \mathrm{~Hz}$ and corresponding voltage trace (top). Right bottom, Time course of EPSC amplitude changes induced by the EPSP-only control protocol (arrow) in layer 1a in a representative cell. Right top, Corresponding averaged EPSCs 4 min before and 15-20 min after EPSP-only control protocol in layer 1a. C, Left, Time course of normalized EPSC amplitude changes in 4-AP induced by the induction protocol based on pairing EPSPs with triple APs $(n=8$; red) and the EPSP-only control protocol $(n=7$; black) in layer 1a. Right, Bar diagram displays the averaged EPSC amplitudes at 15-20 min after different induction protocols normalized to preinduction values. Pairing EPSPs with triple APs in 4-AP for induction in layer 1a leads to significantly larger LTP when compared with EPSPs paired with triple APs in 1a without 4-AP and the EPSP-only control protocol in 4-AP ( ${ }^{*} p<0.05$ and ${ }^{*} p<0.01$, respectively).

termined whether or not these differences in the amplitudes of spine $\mathrm{Ca}^{2+}$ transients also translated into differences in synaptic plasticity induction. First, we used the LTP induction protocol based on pairing EPSPs with three APs but prevented postsynaptic $\mathrm{Ca}^{2+}$ increase with application of $30 \mathrm{~mm}$ intracellular BAPTA. This resulted in a block of LTP, with an EPSP amplitude of $92 \pm$ $5 \%$ of baseline values $(n=7 ; p<0.05)$ (Fig. $8 A, D)$. Another method used for reducing the spine $\mathrm{Ca}^{2+}$ transient was the application of a pairing paradigm with one AP instead of three (Figs. $5 D, 7 B)$. Under these conditions, LTP could not be induced: the EPSC amplitude was $79 \pm 14 \%$ of preinduction baseline values $(n=5)$ (Fig. $8 D)$ and significantly smaller than potentiation levels after the induction with three APs $(p<0.01)$. As a control experiment, EPSPs alone were used for LTP induction (Fig. 8C). The EPSC amplitude was $90 \pm 10 \%$ of the preinduction value $(n=6)$. This result is not significantly different from that after LTP induction with a protocol based on the pairing of an EPSP with a single AP or with three APs in the presence of $30 \mathrm{~mm}$ intracellular BAPTA $(p>0.05)$ (Fig. $8 D$ ). However, the difference was significant when comparing these results with the induction protocol based on pairing EPSPs with three APs in the absence of BAPTA $(p<0.05)$ (Fig. 8D).

We next tested whether or not short high-frequency bursts could be evoked by coincident single-pulse stimulation of layer $1 \mathrm{a}$ and layer $1 \mathrm{~b}$ inputs. Strong perisomatic inhibition, which occurs as a result of extracellular stimulation, resulted in isolated APs. For this reason, we proceeded under conditions of reduced inhibition ( $2 \mu \mathrm{M}$ gabazine). Single subthreshold pulses in layer $1 \mathrm{a}$ and $1 \mathrm{~b}$ were paired at different time intervals. Coincident subthreshold activation of layer 1a and layer $1 b$ at time intervals between -10 and +10 ms resulted in bursts of two to four APs. The first three spikes of the bursts were in the frequency range of $\sim 100 \mathrm{~Hz}$. Longer time intervals $(-100$, $-50,50$, and $100 \mathrm{~ms}$ ) did not result in bursting (Fig. $8 E, D$ ). It is important to note that suprathreshold activation of isolated layer $1 \mathrm{a}$ and layer $1 \mathrm{~b}$ inputs resulted in similar bursts. In neocortical layer 5 pyramidal cells, bursting has also been evoked by pairing an EPSP with a bAP (Larkum et al., 1999b). We also wanted to test whether subthreshold depolarization by a layer 1b EPSP could result in bursting when combined with a somatically evoked spike under conditions of reduced inhibition. Despite the strong effect of coincident subthreshold synaptic activation between -10 and $+10 \mathrm{~ms}$, pairing at similar time intervals did not yield any bursts when an EPSP was paired with a somatically evoked bAP (supplemental Fig. S1, available at www. jneurosci.org as supplemental material).

\section{Discussion}

Dendritic compartmentalization determined by the synaptic input layer

We compared dendritic segments in the proximal associative input layer $1 \mathrm{~b}$ with the distal sensory input layer $1 \mathrm{a}$. In layer $1 \mathrm{a}$, the amplitude of bAP-mediated $\mathrm{Ca}^{2+}$ signals decreased. In neocortical pyramidal cells, this distance-dependent decrease can be overcome by burst firing-mediated distal $\mathrm{Ca}^{2+}$ spikes (Larkum et al., 1999a; Waters et al., 2003; Kampa and Stuart, 2006; Larkum et al., 2007). In layer 2 pyramidal cells of the piriform cortex, we did not observe such a regenerative mechanism, which is comparable with the CA1 pyramidal cell in the hippocampus (for 
A

layer $1 \mathrm{~b} 30 \mathrm{mM}$ BAPTA
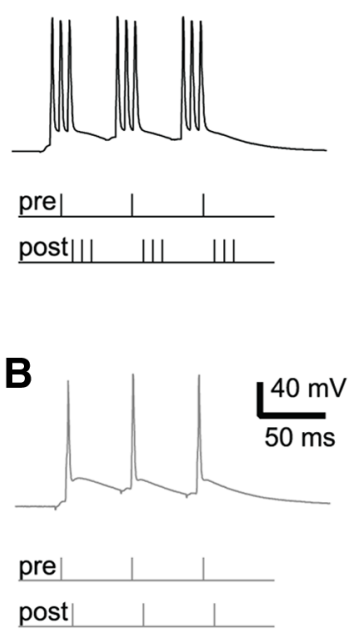

C
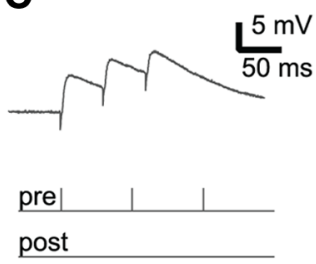

D

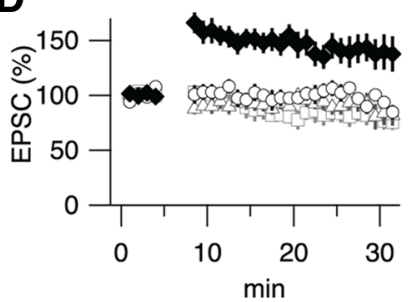

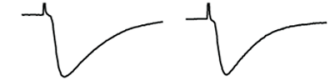
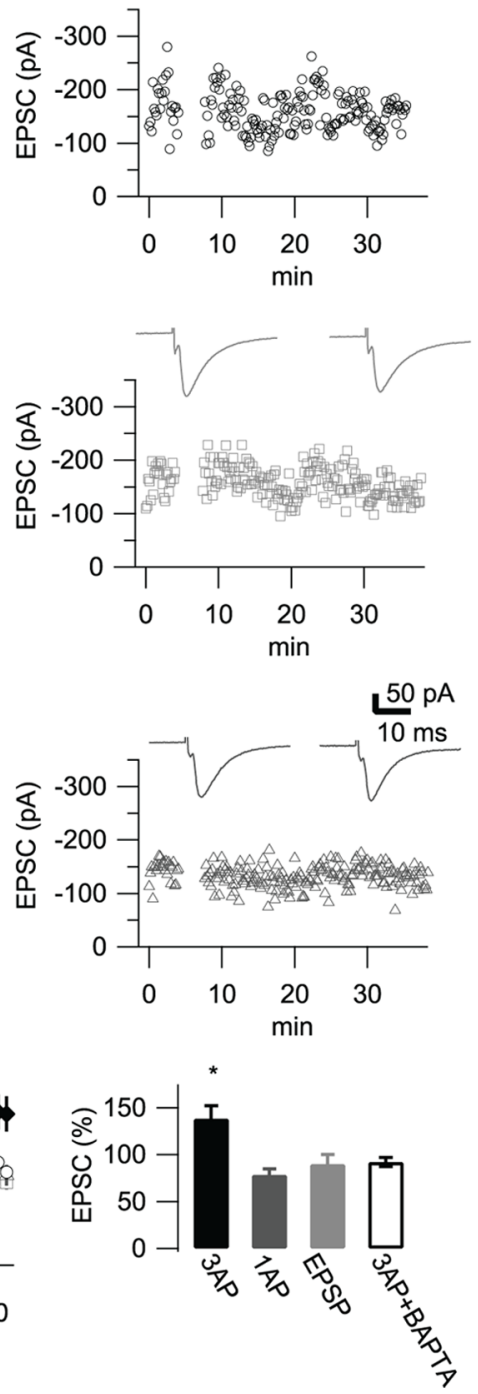

E

pairing layer $1 \mathrm{a}+$ layer $1 \mathrm{~b}$ input

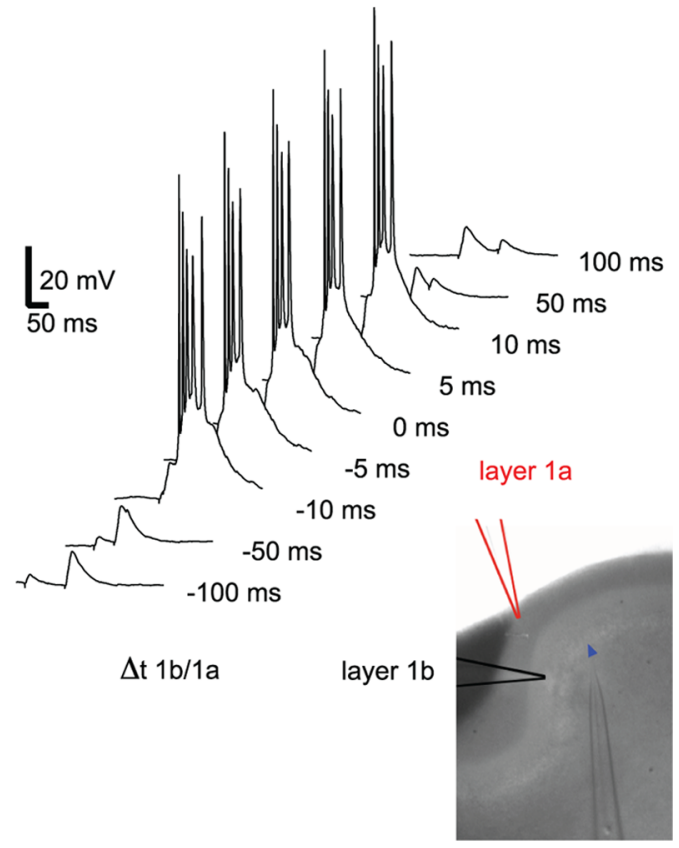

$\mathbf{F}$

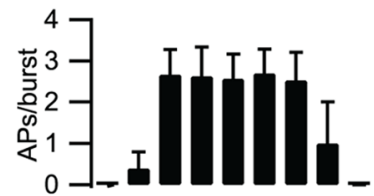

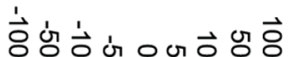

$\Delta \mathrm{t} 1 \mathrm{~b} / 1 \mathrm{a}(\mathrm{ms})$

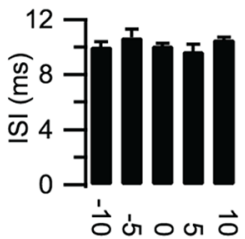

$\Delta \mathrm{t} 1 \mathrm{~b} / 1 \mathrm{a}(\mathrm{ms})$

Figure 8. Pairing EPSPs with postsynaptic single APs does not result in layer 1b LTP. A, Left, Schematic illustration of the LTP induction protocol with 30 mm intracellular BAPTA and voltage trace of a typical induction stimulus (top). Bottom right, Time course of EPSC amplitude changes induced by the induction protocol (arrow) based on pairing EPSPs with three APs in layer $1 \mathrm{~b}$ in a representative cell with $30 \mathrm{~mm}$ intracellular BAPTA. Top right, Corresponding averaged EPSCs 4 min before and $20-25$ min after induction in layer $1 \mathrm{~b}$. $\boldsymbol{B}$, Left, Schematic illustration of the induction protocol consisting of 30 bursts of EPSP triplets at $20 \mathrm{~Hz}$ followed by a single AP $8 \mathrm{~ms}$ after each EPSP and corresponding voltage trace (top). Bottom right, Time course of EPSC amplitude changes induced by the induction protocol (arrow) based on pairing EPSPs with single APs in layer $1 \mathrm{~b}$ in a representative cell. Top right, Corresponding averaged EPSCs 4 min before and $20-25$ min after induction in layer 1b. C, Left, Schematic illustration of the control protocol consisting of 30 bursts of EPSP triplets at $20 \mathrm{~Hz}$ and corresponding voltage trace (top). Bottom right, Time course of EPSC amplitude changes induced by the EPSP-only control protocol (arrow) in layer $1 \mathrm{~b}$ in a representative cell. Top right, Corresponding averaged EPSCs 4 min before and $20-25$ min after administration of the EPSP-only control protocol. D, Left, Time course of normalized EPSC amplitude changes induced by the induction protocol based on pairing EPSPs with three APs ( $n=10$; black diamonds), three APs and intracellular BAPTA ( $n=6$; black circles), single APs ( $n=5$; light gray squares), and the EPSP-only control protocol ( $n=6$; dark gray triangles) in layer $1 \mathrm{~b}$. Right, Bar diagram displays the averaged EPSC amplitudes at $20-25$ min after different induction protocols normalized to preinduction values. LTP based on pairing EPSPs with three APs in layer $1 \mathrm{~b}$ is significantly larger when compared with pairing EPSPs with single APs, the EPSP-only protocol, and the EPSP-three AP protocol with $30 \mathrm{~mm}$ intracellular BAPTA $\left({ }^{*} p<0.05\right)$. $E$, The waterfall plot represents one of six anterior piriform cortex pyramidal cells in which layer 1a and layer $1 \mathrm{~b}$ inputs as indicated in the inset were stimulated with different time intervals. Between -10 (layer $1 \mathrm{~b}$ before layer 1a) and +10 (layer 1a before layer $1 \mathrm{~b}$ ) $\mathrm{ms}$, we observed burst firing of subthreshold signals, which was not present at longer time intervals. $F$, Left, The bar graph indicates the number of APs/burst for the different time intervals ( $n=3$ to 6). Right, The bar graph displays the average interspike intervals (ISI) of the first three spikes in a burst. Four of six cells displayed more than two subsequent spikes.

review, see Sjöström et al., 2008). It is tempting to speculate that the palaeocortex, like its phylogenetic relative, the threelayered amphibian cortex in the turtle (Larkum et al., 2008), does not use supralinear $\mathrm{Ca}^{2+}$ spikes for dendritic signal integration. This would render AP burst-mediated $\mathrm{Ca}^{2+}$ spikes an evolutionary younger mechanism, which is an exclusive property of the neocortex.
A failure of the active spread of the voltage signal mediates the distal attenuation of bAP-mediated $\mathrm{Ca}^{2+}$ transients in CA1 pyramidal cells in the hippocampus and in basal dendrites of layer 5 pyramidal cells. However, there is substantial distal expression of voltage-gated $\mathrm{Ca}^{2+}$ channels. The underlying mechanism for this is the differential distribution of the depolarization-activated A-type $\mathrm{K}^{+}$-channels, which provides an electrical shunt (Hoff- 
man et al., 1997; Kampa and Stuart, 2006; Gasparini et al., 2007). We observed a similar situation in apical dendrites of layer 2 pyramidal cells in the piriform cortex: the pharmacological block of A-type $\mathrm{K}^{+}$conductances resulted in a significant increase in the amplitude of bAP-mediated $\mathrm{Ca}^{2+}$ signals in layer $1 \mathrm{a}$, and we demonstrated that expression levels of Kv4.2 and Kv4.3 (the subunits underlying dendritic A-type conductances) are higher in layer $1 \mathrm{a}$ than in layer $1 \mathrm{~b}$. Finally, failure of voltage propagation is documented using the coincidence detection mechanism of the NMDAR as a reporter (Kampa et al., 2004; Nevian and Sakmann, 2004). This second readout of dendritic depolarization is of special importance, because the distance-dependent amplitude of bAP-mediated $\mathrm{Ca}^{2+}$ signals may be affected by factors different from the spread of the voltage signal.

\section{Spines in different input layers of the apical dendrite constitute distinct plasticity compartments based on integrative dendritic properties}

For LTP induction based on the pairing of presynaptic and postsynaptic activity in mammalian principal neurons, the presynaptic cell must fire milliseconds before the postsynaptic cell (Magee and Johnston, 1997; Markram et al., 1997; Bi and Poo, 1998; Debanne et al., 1998) (for review, see Kampa et al., 2007). Spine $\mathrm{Ca}^{2+}$ transients evoked by these pairing sequences are the elementary building block of associated induction protocols (Nevian and Sakmann, 2006) (for review, see Sjöström et al., 2008). Our data suggest that the electrical properties of layer 2 pyramidal cell dendrites in the piriform cortex result in singlespine $\mathrm{Ca}^{2+}$ signals during LTP induction that differ between layers $1 \mathrm{a}$ and $1 \mathrm{~b}$. Factors determining spine $\mathrm{Ca}^{2+}$ reduction in layer 1a are (1) the failure of the bAP to relieve the $\mathrm{Mg}^{2+}$ block of the NMDAR, and (2) a smaller VGCC-mediated $\mathrm{Ca}^{2+}$ influx. In distal dendrites of neocortical and hippocampal CA1 pyramidal cells, the induction of LTP by pairing EPSPs with subsequent APs is impaired (Golding et al., 2002; Letzkus et al., 2006; Sjöström and Häusser, 2006). Consistent with this, we were only able to induce LTP by pairing EPSPs with subsequent AP bursts in the proximal layer $1 \mathrm{~b}$. This effect on synaptic plasticity underscores the functional relevance of our observation that spine $\mathrm{Ca}^{2+}$ signals reflect an input layer-specific dendritic compartmentalization.

\section{Impairment of distal LTP induction complements previous findings on impaired expression of layer la LTP}

After a brief postnatal critical period, NMDAR-dependent LTP in layer $2 / 3$ cells of the anterior piriform cortex is more pronounced in the associative layer $1 \mathrm{~b}$ than in the sensory layer la synapses (Jung et al., 1990a,b; Kanter and Haberly, 1990; Franks and Isaacson, 2005; Poo and Isaacson, 2007). Looking for a mechanistic explanation for this phenomenon, we observed striking differences between layer $1 \mathrm{a}$ and layer $1 \mathrm{~b}$ in the spine $\mathrm{Ca}^{2+}$ transients underlying LTP induction. These findings constitute a complementary extension of a previously reported mechanism for the expression of LTP. Franks and Isaacson (2005) reported a selective reduction of silent synapses in layer la after a postnatal critical period. However, synapses do not have to be silent to undergo LTP (Matsuzaki et al., 2004; Bagal et al., 2005; Harvey and Svoboda, 2007) (for review, see Kerchner and Nicoll, 2008). It is also important to note that layer 1a LTP can be rescued by low extracellular $\left[\mathrm{Mg}^{2+}\right]$ (Jung et al., 1990b). Low extracellular $\left[\mathrm{Mg}^{2+}\right]$ releases the $\mathrm{Mg}^{2+}$ block of the NMDAR, which compensates for a reduction in layer 1a depolarization and results in elevated postsynaptic $\left[\mathrm{Ca}^{2+}\right]$ during LTP induction. When blocking A-type $\mathrm{K}^{+}$channels with 4 -AP, pairing EPSPs with bAPs readily induced layer 1a LTP. This was not possible in controls using EPSPs without bAPs, indicating that the enhanced AP backpropagation elevates postsynaptic $\mathrm{Ca}^{2+}$, which rescues layer 1a LTP.

LTP induction is a complex phenomenon with a plethora of synapse-specific underlying mechanisms. It is conceivable that there are additional differences in the molecular machinery of layer $1 \mathrm{a}$ and layer $1 \mathrm{~b}$ presynaptic and postsynaptic architecture that result in different levels of induction and expression of synaptic plasticity. Synapses projecting to layer $1 \mathrm{a}$ and layer $1 \mathrm{~b}$ inputs have entirely different origins (mitral/tufted cells in the olfactory bulb for layer $1 \mathrm{a}$ and piriform cortex pyramidal cells, associative connections from other cortical areas for layer 1b). Moreover, electron microscopic analysis of layer 1a and layer 1b synapses indicated striking morphological differences (Haberly and Behan, 1983).

Coincidence detection of presynaptic and postsynaptic firing by the NMDAR also reports the cellular output mode In associative layer $1 \mathrm{~b}$ spines of piriform cortex layer 2 pyramidal cells, postsynaptic bursting-mediated $\mathrm{Ca}^{2+}$ signals are a prerequisite for LTP induction. Pairing EPSPs with subsequent bursts of APs has also been shown to be a prerequisite for LTP induction in pyramidal cells in CA1 and the neocortex (Pike et al., 1999; Kampa et al., 2006; Letzkus et al., 2006; Nevian and Sakmann, 2006). The most obvious reason for the requirement of bursting for LTP induction is the additional $\mathrm{Ca}^{2+}$ provided by a larger degree of VGCC activation. On the level of single-spine EPSP-AP integration, our data suggests an additional mechanism: one AP does not fully relieve the $\mathrm{Mg}^{2+}$ block of the NMDAR, resulting in relatively small supralinearity ratios. Additional APs increase the supralinear $\mathrm{Ca}^{2+}$ influx mediated by the NMDAR. The gain of this coincidence detector is therefore set in a range that also reports the output mode of the cell at the level of single-spine integration.

\section{Functional implications for learning in the piriform cortex network}

The lack of LTP in layer 1a synapses after the critical period has been assumed to result in the hardwiring of sensory olfactory bulb inputs to the piriform cortex (Franks and Isaacson, 2005). We document that this concept of hardwiring is supported by a functional compartmentalization of the apical dendritic tree, based on intrinsic electrical properties that influence plasticityinducing spine $\mathrm{Ca}^{2+}$ transients. This underscores the importance of dendritic excitability for LTP induction (Magee and Johnston, 1997) (for review, see Sjöström et al., 2008).

The potentiation of associative EPSPs depends not only on the strength of the excitatory inputs but also on the output mode of the cell. The output mode is regulated by the balance of excitation and inhibition resulting from synaptic and intrinsic currents. Luna and Schoppa (2008) observed a strong perisomatic inhibitory control exerted by single GABAergic interneurons. One could envision a mechanism in which GABAergic interneurons control the plasticity of excitatory synapses by controlling the output mode of the cell. The net result is a high threshold for the amplification of associative synapses, a mechanism promoting network stability. Interestingly, olfactory rule learning, a behavioral state that corresponds to enhanced learning capability, is associated with a temporary increase in general intrinsic excitability in the piriform cortex (for review, see Barkai and Saar, 2001). Neuromodulation by cholinergic projections promotes this increase in excitability and learning capability (Saar et al., 
2001). One could assume a connection between enhanced learning and facilitated synaptic strengthening. A previous enhancement of burst firing during rule learning would then increase the probability of synaptic strengthening in associative layer $1 \mathrm{~b}$ networks.

A-type $\mathrm{K}^{+}$channel-mediated currents are dynamically regulated by LTP, inducing synaptic activation patterns. The NMDAR-dependent enhancement of synaptic strength results in a hyperpolarized shift of A-type $\mathrm{K}^{+}$channel inactivation (Frick and Johnston, 2005) and the internalization of Kv4.2 subunits (Kim et al., 2007). It is interesting to speculate that similar mechanisms could facilitate layer 1a LTP. In addition, neuromodulators such as acetylcholine or noradrenalin reduce A-type $\mathrm{K}^{+}$ channel-mediated currents, promoting the backpropagation of APs into distal dendritic compartments (Hoffman and Johnston, 1999). One could therefore envision a softening of the hardwiring of layer 1a connections that occurs after the critical period and during the learning-associated neuromodulatory network states gating layer la synaptic plasticity.

\section{References}

Bagal AA, Kao JP, Tang CM, Thompson SM (2005) Long-term potentiation of exogenous glutamate responses at single dendritic spines. Proc Natl Acad Sci U S A 102:14434-14439.

Barkai E, Saar D (2001) Cellular correlates of olfactory learning in the rat piriform cortex. Rev Neurosci 12:111-120.

Bi GQ, Poo MM (1998) Synaptic modifications in cultured hippocampal neurons: dependence on spike timing, synaptic strength, and postsynaptic cell type. J Neurosci 18:10464-10472.

Birnbaum SG, Varga AW, Yuan LL, Anderson AE, Sweatt JD, Schrader LA (2004) Structure and function of Kv4-family transient potassium channels. Physiol Rev 84:803-833.

Debanne D, Gähwiler BH, Thompson SM (1998) Long-term synaptic plasticity between pairs of individual CA3 pyramidal cells in rat hippocampal slice cultures. J Physiol 507:237-247.

Franks KM, Isaacson JS (2005) Synapse-specific downregulation of NMDA receptors by early experience: a critical period for plasticity of sensory input to olfactory cortex. Neuron 47:101-114.

Frick A, Johnston D (2005) Plasticity of dendritic excitability. J Neurobiol 64:100-115.

Gasparini S, Losonczy A, Chen X, Johnston D, Magee JC (2007) Associative pairing enhances action potential back-propagation in radial oblique branches of CA1 pyramidal neurons. J Physiol 580:787-800.

Golding NL, Staff NP, Spruston N (2002) Dendritic spikes as a mechanism for cooperative long-term potentiation. Nature 418:326-331.

Haberly L, Behan M (1983) Structure of the piriform cortex of the opossum. III. Ultrastructural characterization of synaptic terminals of association and olfactory bulb afferent fibers. J Comp Neurol 219:448-460.

Harvey CD, Svoboda K (2007) Locally dynamic synaptic learning rules in pyramidal neuron dendrites. Nature 450:1195-1200.

Higley MJ, Sabatini BL (2008) Calcium signaling in dendrites and spines: practical and functional considerations. Neuron 59:902-913.

Hoffman DA, Johnston D (1999) Neuromodulation of dendritic action potentials. J Neurophysiol 81:408-411.

Hoffman DA, Magee JC, Colbert CM, Johnston D (1997) $\mathrm{K}^{+}$channel regulation of signal propagation in dendrites of hippocampal pyramidal neurons. Nature 387:869-875.

Jung MW, Larson J, Lynch G (1990a) Role of NMDA and non-NMDA receptors in synaptic transmission in rat piriform cortex. Exp Brain Res $82: 451-455$.

Jung MW, Larson J, Lynch G (1990b) Long-term potentiation of monosynaptic EPSPs in rat piriform cortex in vitro. Synapse 6:279-283.

Kampa BM, Stuart GJ (2006) Calcium spikes in basal dendrites of layer 5 pyramidal neurons during action potential bursts. J Neurosci 26: $7424-7432$.

Kampa BM, Clements J, Jonas P, Stuart GJ (2004) Kinetics of $\mathrm{Mg}^{2+}$ unblock of NMDA receptors: implications for spike-timing dependent synaptic plasticity. J Physiol 556:337-345.
Kampa BM, Letzkus JJ, Stuart GJ (2006) Requirement of dendritic calcium spikes for induction of spike-timing-dependent synaptic plasticity. J Physiol 574:283-290.

Kampa BM, Letzkus JJ, Stuart GJ (2007) Dendritic mechanisms controlling spike-timing-dependent synaptic plasticity. Trends Neurosci $30: 456-463$.

Kanter ED, Haberly LB (1990) NMDA-dependent induction of long-term potentiation in afferent and association fiber systems of piriform cortex in vitro. Brain Res 525:175-179.

Kerchner GA, Nicoll RA (2008) Silent synapses and the emergence of a postsynaptic mechanism for LTP. Nat Rev Neurosci 9:813-825.

Kim J, Jung SC, Clemens AM, Petralia RS, Hoffman DA (2007) Regulation of dendritic excitability by activity-dependent trafficking of the A-type $\mathrm{K}^{+}$channel subunit Kv4.2 in hippocampal neurons. Neuron 54:933-947.

Koester HJ, Sakmann B (1998) Calcium dynamics in single spines during coincident pre- and postsynaptic activity depend on relative timing of back-propagating action potentials and subthreshold excitatory postsynaptic potentials. Proc Natl Acad Sci U S A 95:9596-9601.

Larkum ME, Kaiser KM, Sakmann B (1999a) Calcium electrogenesis in distal apical dendrites of layer 5 pyramidal cells at a critical frequency of back-propagating action potentials. Proc Natl Acad Sci USA 96:14600-14604.

Larkum ME, Zhu JJ, Sakmann B (1999b) A new cellular mechanism for coupling inputs arriving at different cortical layers. Nature 398:338-341.

Larkum ME, Waters J, Sakmann B, Helmchen F (2007) Dendritic spikes in apical dendrites of neocortical layer $2 / 3$ pyramidal neurons. J Neurosci 27:8999-9008.

Larkum ME, Watanabe S, Lasser-Ross N, Rhodes P, Ross WN (2008) Dendritic properties of turtle pyramidal neurons. J Neurophysiol 99:683-694.

Letzkus JJ, Kampa BM, Stuart GJ (2006) Learning rules for spike timingdependent plasticity depend on dendritic synapse location. J Neurosci 26:10420-10429.

Luna VM, Schoppa NE (2008) GABAergic circuits control input-spike coupling in the piriform cortex. J Neurosci 28:8851-8859.

Magee JC, Johnston D (1997) A synaptically controlled, associative signal for Hebbian plasticity in hippocampal neurons. Science 275:209-213.

Markram H, Lübke J, Frotscher M, Sakmann B (1997) Regulation of synaptic efficacy by coincidence of postsynaptic APs and EPSPs. Science 275:213-215

Matsuzaki M, Honkura N, Ellis-Davies GC, Kasai H (2004) Structural basis of long-term potentiation in single dendritic spines. Nature 429:761-766.

Mokin M, Keifer J (2006) Quantitative analysis of immunofluorescent punctate staining of synaptically localized proteins using confocal microscopy and stereology. J Neurosci Methods 157:218-224.

Nevian T, Sakmann B (2004) Single spine $\mathrm{Ca}^{2+}$ signals evoked by coincident EPSPs and backpropagating action potentials in spiny stellate cells of layer 4 in the juvenile rat somatosensory barrel cortex. J Neurosci 24:1689-1699.

Nevian T, Sakmann B (2006) Spine $\mathrm{Ca}^{2+}$ signaling in spike-timingdependent plasticity. J Neurosci 26:11001-11013.

Neville KR, Haberly LB (2004) Olfactory cortex. In: The synaptic organization of the brain, Ed 5 (Shepherd GM, ed), pp 415-454. New York: Oxford UP.

Pérez-Garci E, Gassmann M, Bettler B, Larkum ME (2006) The GABAB1b isoform mediates long-lasting inhibition of dendritic $\mathrm{Ca}^{2+}$ spikes in layer 5 somatosensory pyramidal neurons. Neuron 50:603-616.

Pike FG, Meredith RM, Olding AW, Paulsen O (1999) Rapid report: postsynaptic bursting is essential for "Hebbian" induction of associative long-term potentiation at excitatory synapses in rat hippocampus. J Physiol 518:571-576.

Poo C, Isaacson JS (2007) An early critical period for long-term plasticity and structural modification of sensory synapses in olfactory cortex. J Neurosci 27:7553-7558.

Poo C, Isaacson JS (2009) Odor representations in olfactory cortex: "sparse" coding, global inhibition, and oscillations. Neuron 62:850-861.

Saar D, Grossman Y, Barkai E (2001) Long-lasting cholinergic modulation underlies rule learning in rats. J Neurosci 21:1385-1392.

Sabatini BL, Oertner TG, Svoboda K (2002) The life cycle of $\mathrm{Ca}^{2+}$ ions in dendritic spines. Neuron 33:439-452. 
Shepherd GM (2004) Introduction to synaptic circuits. In: The synaptic organization of the brain, Ed 5 (Shepherd GM, ed), pp 1-39. New York: Oxford UP.

Sjöström PJ, Häusser M (2006) A cooperative switch determines the sign of synaptic plasticity in distal dendrites of neocortical pyramidal neurons. Neuron 51:227-238.

Sjöström PJ, Rancz EA, Roth A, Häusser M (2008) Dendritic excitability and synaptic plasticity. Physiol Rev 88:769-840.

Suzuki N, Bekkers JM (2006) Neural coding by two classes of principal cells in the mouse piriform cortex. J Neurosci 26:11938-11947.
Tang AC, Hasselmo ME (1994) Selective suppression of intrinsic but not afferent fiber synaptic transmission by baclofen in the piriform (olfactory) cortex. Brain Res 659:75-81.

Waters J, Larkum M, Sakmann B, Helmchen F (2003) Supralinear $\mathrm{Ca}^{2+}$ influx into dendritic tufts of layer $2 / 3$ neocortical pyramidal neurons in vitro and in vivo. J Neurosci 23:8558-8567.

Wilson DA (1998) Habituation of odor responses in the rat anterior piriform cortex. J Neurophysiol 79:1425-1440.

Yuste R, Denk W (1995) Dendritic spines as basic functional units of neuronal integration. Nature 375:682-684. 